Pure and Applied Mathematics Quarterly

Volume 3, Number 1

(Special Issue: In honor of

Robert MacPherson, Part 3 of 3 )

323-346, 2007

\title{
On the Fibres of a Toroidal Resolution
}

\author{
Arvind N. Nair \\ To R. MacPherson

\section{INTRODUCTION}

The quotient $M=\Gamma \backslash D$ of a Hermitian symmetric domain by an arithmetic group of automorphisms is a quasiprojective algebraic variety. Varieties like this, variously called modular or locally symmetric or arithmetic varieties, play an important role in representation theory and arithmetic. Many naturally occurring arithmetic varieties are noncompact, and the study of their compactifications has a long history. The variety $M$ has a canonical embedding in a projective space given by certain automorphic forms for $\Gamma$ (essentially sections of a power of the canonical bundle); the closure $M^{*}$ in this embedding is the minimal compactification of Satake and Baily-Borel. It is a normal variety which usually has complicated singularities at the boundary and any smooth compactification in which $M$ is the complement of a normal-crossings divisor dominates it. There is no canonical smooth compactification of $M$ in general, but in [1], Mumford et al. showed how to desingularize $M^{*}$ using an extra choice. Given a suitable $\Gamma$ admissible rational polyhedral cone decomposition $\Sigma$ (the notion is recalled in $\S \S 2,4$ ), the method produces a smooth projective toroidal compactification $M^{\Sigma}$ in which the complement of $M$ is a divisor with simple normal crossings. There is a morphism

$$
\pi: M^{\Sigma} \rightarrow M^{*}
$$

extending the identity on $M$. It is of interest in various questions to study the fibres of $\pi$. In this paper I want to describe a homological property of these fibres when the Q-rank of $M$ is one (so that $M^{*}-M$ is the quotient of a smooth variety by a finite group). I shall assume always that $\Gamma$ is neat (which can always be achieved by passing to a subgroup of finite index), so that $M^{*}-M$ is smooth. In this introduction it will be further assumed that $M^{*}-M$ consists of points (i.e. $D$ is a $\mathbb{Q}$-rank one tube domain with cusps). Examples include Hilbert modular varieties and arithmetic varieties associated to $\mathbb{Q}$-rank one forms of $\mathrm{SO}(2, n)$.

Received November 9, 2005. 
A fibre of $\pi: M^{\Sigma} \rightarrow M^{*}$ at a cusp $s \in M^{*}-M$ is the geometric quotient

$$
\pi^{-1}(s)=\Lambda \backslash \mathcal{D}
$$

of a certain scheme $\mathcal{D}$ by a free action of an infinite discrete group $\Lambda$. The scheme $\mathcal{D}$ is not usually of finite type, but each irreducible component is smooth and of finite type, and $\mathcal{D}$ has normal crossings. There is a natural ind-variety structure on $\mathcal{D}$ (as we show here). The simplest example is that of Hilbert modular surfaces, where the preimage of a cusp of $M^{*}$ is a cycle of rational curves: the quotient of an infinite chain of projective lines joined "end-to-end" (our $\mathcal{D}$ ) by an infinite cyclic group (our $\Lambda$ ). In general, there is a morphism $\mathcal{D} \rightarrow A$ to an abelian variety which on each irreducible component of $\mathcal{D}$ restricts to a smooth projective toric fibration over $A$. The group $\Lambda$ is an arithmetic subgroup of the automorphism group of a self-adjoint homogeneous cone $C$ (e.g. $\Lambda$ could be $\mathbb{Z}^{d}$ or $\mathrm{SL}(n, \mathbb{Z})$ or an arithmetic group in $\left.\mathrm{SO}(n, 1)\right)$.

The ind-variety $\mathcal{D}$ comes with an embedding in a certain smooth scheme $y \rightarrow A$ locally of finite type; indeed, $y$ is a relative torus embedding of a relative torus $\mathcal{T} \rightarrow A$ using the cone system $\Sigma$, and $\mathcal{D}$ is the complement of the dense torus $\mathcal{T}$ in $y$. The action of $\Lambda$ comes from one on $y$, but it is not proper, so that the quotient $\Lambda \backslash y$ exists only as an analytic space and not as a scheme. There is an analytic map $\Lambda \backslash y \rightarrow M^{\Sigma}$ identifying $\Lambda \backslash \mathcal{D}$ with the fibre and it is an analytic isomorphism locally along $\Lambda \backslash \mathcal{D}$. (Indeed, $M^{\Sigma}$ is actually constructed in [1] by gluing together certain analytic neighbourhoods of $\Lambda \backslash \mathcal{D}$ in $\Lambda \backslash y$ for the various cusps of $M^{*}$.) The main result of this note is that $\mathcal{D} \hookrightarrow y$ is a homology isomorphism (cf. Thm 3.2 when $A$ is trivial, Thm 4.1 in general), i.e.

$$
\mathrm{H}_{*}(\mathcal{D})=\mathrm{H}_{*}(y) .
$$

Since $\Lambda$ acts freely on $\mathcal{D}$, it follows that there is a natural isomorphism

$$
\mathrm{H}_{*}(\Lambda \backslash \mathcal{D})=\mathrm{H}_{*}^{\Lambda}(y)
$$

giving a description of the homology of the fibre as the $\Lambda$-equivariant homology of the smooth scheme $y$. This suggests that the "quotient" of the smooth scheme $y$ by $\Lambda$ should be thought of as an algebraic version of a regular neighbourhood of the fibre in $M^{\Sigma}$. A consequence of the isomorphism $\mathrm{H}_{i}(\mathcal{D}) \cong \mathrm{H}_{i}(y)$ is that this group is pure of weight $-i$, i.e. the ind-variety $\mathcal{D}$ is pure. (This is Corollary 3.2 when $A$ is trivial; note that this is only obvious in the simplest case of Hilbert modular surfaces.) Roughly speaking, $\mathcal{D}$ satisfies the valuative criterion for properness, so that $\mathrm{H}_{i}(\mathcal{D})$ has weights $\geq-i$, while $y$ is smooth, so that $\mathrm{H}_{i}(y)$ has weights $\leq-i$. This purity has pleasant consequences: the spectral sequence computing $\mathrm{H}_{*}(\Lambda \backslash \mathcal{D})=\mathrm{H}_{*}^{\Lambda}(y)$ in terms of $\Lambda$ homology of $\mathrm{H}_{*}(\mathcal{D}) \cong \mathrm{H}_{*}(y)$ degenerates at $\mathrm{E}_{2}$ and the limit filtration is (with a shift) the weight filtration (Cor. 3.4, Thm 4.1). So one has an expression for the graded pieces of the weight filtration on the fibre homology in terms of group homology and $y .\left(^{1}\right)$

\footnotetext{
${ }^{1}$ There is a formal resemblance between the fibres studied here and the varieties appearing in [5] in the context of the "fundamental lemma": In each case one has a quotient of a ind-variety by a discrete group. Here, the discrete group $\Lambda$ (which is typically like $\operatorname{GL}(n, \mathbb{Z})$ ) is complicated, while the ind-variety $\mathcal{D}$ is
} 
Another consequence of purity (to which attention was drawn in [4]) is that the torus action on $\mathcal{D}$ is equivariantly formal. It follows that the equivariant cohomology is localized to the fixed-point locus and has a description in terms of the fixed point locus and one-dimensional (over $A$ ) orbits. This is mentioned in 3.6 and 4.5, a fuller treatment being left for another occasion. The purity statement also has consequences for the Hodge theory of the exceptional divisor $\pi^{-1}\left(M^{*}-M\right)$, which we mention in 4.6.

The essential tool in proving these results is an enumeration of the top-dimensional cones in $\Sigma$. The cone system $\Sigma$ is a decomposition of the open self-adjoint homogeneous cone $C$ into rational polyhedral cones. For example, if $M$ is a Hilbert modular surface associated to a real quadratic field $F$, the cone $C$ is the convex hull in $F \otimes_{\mathbb{Q}} \cong \mathbb{R}^{2}$ of the set of totally positive elements of $F$ and $\Sigma$ is a decomposition of $C \cong \mathbb{R}_{+}^{2}$ into rational sectors, invariant under the action of a subgroup $\Lambda \cong \mathbb{Z}$ of the group of totally positive units of $F$. In general, the fan $\Sigma$ consists of cones on the faces of an unbounded locally polyhedral convex set $P$ (a $\Lambda$-polyhedral cocore in the terminology of [1, Chp. II]), the convexity of $P$ being responsible for the projectivity of $M^{\Sigma}$. The idea of line shellings from the theory of convex polytopes allows us to enumerate the facets of $P$, and hence the top-dimensional cones of $\Sigma$, in a nice way (Prop. 2.5). This gives the ind-variety structure of $\mathcal{D}$ and defines filtrations of $\mathcal{D}$ and $y$ which allow for an inductive proof that $\mathrm{H}_{*}(\mathcal{D}) \cong \mathrm{H}_{*}(y)$ using elementary facts about the topology of torus embeddings.

The methods used here to study the fibres of $\pi$ are applicable if the $\mathbb{Q}$-rank is $\geq 2$, but the results have to be reformulated (cf. 3.10 for some comments).

The contents of this article are as follows: In $\S 1$ the necessary properties of selfadjoint homogeneous cones are recalled from [1]. In $\S 2$ the construction of nice polyhedral decompositions $\Sigma$ of such cones is recalled, following [1], and the enumeration of top-dimensional cones of such $\Sigma$ mentioned above is proved. (No assumption is made on the $\mathbb{Q}$-rank in these sections, but the simplifications in the case of $\mathbb{Q}$-rank one are indicated.) From $\S 3$ we assume that the $\mathbb{Q}$-rank is one. In $\S 3$ the enumeration from $\S 2$ is used to prove the main theorem when the abelian variety $A$ is trivial. Then weights are brought in and various consequences of purity indicated, under the same assumption. General $\mathbb{Q}$-rank one arithmetic varieties (where $A$ is nontrivial) are treated in $\S 4$.

It is a pleasure to dedicate this article to R. MacPherson on his sixtieth birthday. I hope he finds the mix of topics - convexity, toric geometry, arithmetic groups - appealing.

I thank M. Goresky and E. Looijenga for discussions related to this material and the referee for useful remarks.

simple (its homology is pure). In [5], the discrete group is simple $\left(\cong \mathbb{Z}^{d}\right)$, but the ind-variety, an affine Springer fibre, is complicated. In particular, the conjecture that they are pure appears to be difficult. 


\section{Self-Adjoint homogeneous CONES}

This section contains background material on cones and their geometry. The key fact used later is Lemma 1.2.

1.1. Self-adjoint homogeneous cones. We start a real vector space $V$ of finite dimension $N$ and a nondegenerate open cone

$$
C \subset V
$$

i.e. an open subset invariant under dilations by $\mathbb{R}_{+}$which contains no line in its closure. The (connected) automorphism group of the cone is

$$
\operatorname{Aut}(C)^{0}:=\{g \in \mathrm{GL}(V): g C=C\}^{0} .
$$

The cone is homogeneous if $\operatorname{Aut}(C)^{0}$ acts transitively on $C$. It is self-adjoint if there is an inner product $\langle$,$\rangle on V$ such that $C$ is identified with its dual cone $C^{*}=\{v \in V$ : $\langle v, w\rangle>0 \forall w \in \bar{C}, w \neq 0\}$. A self-adjoint cone is convex.

Let $C \subset V$ be self-adjoint and homogeneous. The automorphism group is a Lie subgroup of GL $(V)$, stable under transpose, and therefore reductive. The isotropy subgroup of any point in $C$ is a maximal compact subgroup, and so $C$ is identified with the symmetric space of $\operatorname{Aut}(C)^{0}$. (Conversely, if a connected reductive Lie group acts on a vector space with an open orbit at points of which the isotropy subgroups are maximal compact, then the orbit is a self-adjoint homogeneous cone.)

Let $\mathfrak{g}$ be the Lie algebra of $\operatorname{Aut}(C)^{0}$. Fix a basepoint $e \in C$ and let $K$ be the isotropy subgroup at $e$. The Cartan decomposition $\mathfrak{g}=\mathfrak{k}+\mathfrak{p}$ given by $K$ fixes an isomorphism

$$
\mathfrak{p} \rightarrow V \quad \text { by } X \mapsto X e
$$

(here $X \in \mathfrak{g}$ acts on $V$ via $\mathfrak{g} \subset \operatorname{End}(V)$ ). For a maximal abelian subspace $\mathfrak{a} \subset \mathfrak{p}$ let $A=e^{\mathfrak{a}}$. Then $C=K A e$.

Let $V_{\mathbb{Q}} \subset V$ be a rational structure. The cone $C$ is rational if there is a $\mathbb{Q}$-algebraic group

$$
G \subset \operatorname{GL}\left(V_{\mathbb{Q}}\right) \quad \text { with } G(\mathbb{R})^{0}=\operatorname{Aut}(C)^{0},
$$

i.e. the automorphism group is, up to connected components, the real points of a $\mathbb{Q}$ algebraic group. This will be assumed to be the case from now on, and we will fix, once and for all, a rational basepoint $e \in C \cap V_{\mathbb{Q}}$. It is known that the inner product on $V$ (with respect to which $C$ is self-dual) may be chosen to be rational.

For $k=\mathbb{Q}$ or $\mathbb{R}$, a cone is $k$-irreducible if it cannot be written as a sum $C=C_{1}+C_{2}$ for a $k$-rational decomposition $V=V_{1} \oplus V_{2}$ and $k$-rational self-adjoint homogeneous cones $C_{i} \subset V_{i}$. An equivalent condition is that $V$ is irreducible for $G$ over $k$ (p. 87 of [1]). In this situation the $k$-split centre of $G$ is one-dimensional and acts by dilations on $V$. Any $k$-rational cone $C$ is a sum of irreducible ones. The $\mathbb{R}$-irreducible cones have 
been classified (cf. [1, p. 63] for the list, which includes four infinite families plus one exceptional cone).

1.2. Jordan algebra. A Jordan algebra is a finite-dimensional commutative algebra in which the product $a \cdot b$ satisfies $a^{2} \cdot(b \cdot a)=\left(a^{2} \cdot b\right) \cdot a$. It is Euclidean if $a^{2}+b^{2}=0$ implies that $a=b=0$.

It is a basic fact ([1, p. 76]) that for a rational self-adjoint homogeneous cone $C \subset V$ as in the previous paragraph, there is a Euclidean Jordan algebra structure on $V$, with $e$ as unit, which is rational over $\mathbb{Q}$. Let $T_{a} \in \mathfrak{p}$ be the element mapped to $a \in V$ under $\mathfrak{p} \cong V$, i.e. $T_{a} e=a$. The Jordan algebra structure satisfies $T_{a}(b)=a \cdot b$ for all $b \in V$. The cone is $C=\left\{x^{2}: x \in V\right.$ is invertible $\}$ and its closure is $\bar{C}=\left\{x^{2}: x \in V\right\}$. The inner product in $V$ can be taken to be $\langle x, y\rangle=$ trace of left multiplication by $x \cdot y$ on $V$. (Note that this is $\mathbb{Q}$-rational.)

Two idempotents $e_{1}, e_{2} \in V$ (for the Jordan algebra structure), are orthogonal if $e_{1} \cdot e_{2}=0$. An idempotent is minimal if it cannot be written as the sum of mutually orthogonal idempotents. A collection of idempotents $e_{1}, \ldots, e_{r}$ is complete if $\sum_{i} e_{i}=$ $e$. A maximal collection of mutually orthogonal idempotents is complete. Let $\left\{e_{i}\right\}$ be such a collection. Let $x_{i} \in \mathfrak{p}$ be defined by $x_{i}=2 T_{e_{i}}$, (i.e. $x_{i} e=2 e_{i}$ in the action of $\mathfrak{p}$ on $V$ ). Then $\mathfrak{a}=\sum_{i} \mathbb{R} x_{i}$ is a maximal abelian subspace of $\mathfrak{p}$, and every such subspace arises from a complete set of mutually orthogonal minimal idempotents in this way (cf. p. 89 of [1]). From the description of the cone as invertible squares, one sees that $C \cap \sum_{i} \mathbb{R}_{i}=A e=\sum_{i} \mathbb{R}_{+} e_{i}$. In other words, the orbit $A e$ is open in its linear span, which is a $e \subset V$, and $\mathfrak{a} e \cap C=A e$ (cf. Prop. 14 on p. 104 of [1]).

Fix a maximal collection of mutually orthogonal idempotents $e_{1}, \ldots, e_{r}$. Let $x_{1}^{*}, \ldots, x_{r}^{*}$ be the basis of $\mathfrak{a}^{*}$ dual to the corresponding basis $x_{1}, \ldots, x_{r}$ of $\mathfrak{a}$. They will be considered as characters on $A$ via the logarithm isomorphism $\log : A \rightarrow \mathfrak{a}$, i.e. $x_{i}^{*}\left(e^{y}\right)=x_{i}^{*}(y)$ for $y \in \mathfrak{a}$.

Lemma 1.1. $\left.\operatorname{det}\right|_{A}=\left(x_{1}^{*}\right)^{\lambda_{1}}\left(x_{2}^{*}\right)^{\lambda_{2}} \ldots\left(x_{r}^{*}\right)^{\lambda_{r}}$ where $\lambda_{i}>0$ for all $i$.

Proof. First assume that $C$ is $\mathbb{R}$-irreducible. The space $V$ has a (Peirce) decomposition

$$
V=\bigoplus_{1 \leq i \leq j \leq r} V_{i j}
$$

where

$$
\begin{aligned}
V_{i i} & =\left\{v \in V: e_{i} \cdot v=v\right\} \\
V_{i j} & =\left\{v \in V: e_{i} \cdot v=e_{j} \cdot v=v / 2\right\} .
\end{aligned}
$$

If $k \neq i, j$ then $e_{k} \cdot v=0$ for $v \in V_{i j}$. For $i \neq j$, the dimension of $V_{i j}$ is a number $d$ which depends only on the cone, i.e. it is independent of $i$ and $j$; the dimension of $V_{i i}$ is one. (These facts are proved in pp. 92-97 of [1].) This gives $N=r+d r(r-1) / 2$. The trace of left multiplication by $e_{i}$ on $V$ is then $1+d(r-1) / 2=N / r$. Since $x_{i}=2 T_{e_{i}}$ we 
have $\operatorname{det}\left(e^{x_{i}}\right)=e^{2 \operatorname{tr}\left(T_{e_{i}}\right)}=e^{2 N / r}$. Then $\left.\operatorname{det}\right|_{A}=\left(x_{1}^{*} x_{2}^{*} \ldots x_{r}^{*}\right)^{2 N / r}$. The general case follows by writing the cone as a sum of irreducible cones and applying this calculation to each one.

Let

$$
G_{1}:=\operatorname{ker}\left(\left.\operatorname{det}\right|_{G}\right)
$$

This is a rational algebraic group, and the symmetric space of $G_{1}(\mathbb{R})^{0}$ is the quotient $C / \mathbb{R}_{+}$of $C$ by dilations. The subgroup $A_{1} \subset G_{1}(\mathbb{R})^{0}$ (i.e. the connected real points of a maximal $\mathbb{R}$-split torus in $G_{1}$ ) is the subgroup $\operatorname{ker}\left(\operatorname{det} \mid{ }_{A}\right)$ of $A$, for which the lemma gives the explicit equation $\prod_{i} t_{i}^{\lambda_{i}}=1$ (in the preferred coordinates on $A$ ). Similarly, $\mathfrak{a}_{1} \subset \mathfrak{a}$ is given by $\Sigma_{i} \lambda_{i} t_{i}=0$.

1.3. Sublevel sets of the characteristic function. Fix a translation-invariant measure $d y$ on $V$. The characteristic function $\varphi: C \rightarrow \mathbb{R}_{+}$of the cone is defined by

$$
\varphi(x):=\int_{C} e^{-\langle x, y\rangle} d y .
$$

It is canonical up to the choice of measure $d y$, i.e. up to a constant. Let us normalize it by requiring $\varphi(e)=1$. The following properties of $\varphi$ are found in [1, p. 57ff]:

(i) $\varphi(g x)=\operatorname{det}(g)^{-1} \varphi(x)$ for $g \in G(\mathbb{R})^{0}, x \in C$. In particular, $\varphi(\lambda x)=$ $\lambda^{-N} \varphi(x)$ for $\lambda \in \mathbb{R}_{+}$. It follows that $\varphi(x) d x$ is a $G(\mathbb{R})^{0}$-invariant measure on $C, \varphi$ is $G_{1}(\mathbb{R})^{0}$-invariant, and its level sets are $G_{1}(\mathbb{R})^{0}$-orbits. By our normalization we have $\varphi(g e)=\operatorname{det}(g)^{-1}$ for $g \in G_{1}(\mathbb{R})^{0}$.

(ii) $\varphi: C \rightarrow \mathbb{R}_{+}$is strictly convex, i.e. $\varphi(t x+(1-t) y)<t \varphi(x)+(1-t) \varphi(y)$ for $x, y \in C, t \in(0,1)$. In particular its sublevel sets are convex.

Fix a point $y \in C$ and consider its $G_{1}(\mathbb{R})^{0}$-orbit:

$$
S:=G_{1}(\mathbb{R})^{0} y
$$

It is a level set of $\varphi$ (by (i)) and $\mathbb{R}_{\geq 1} S$ is a sublevel set of $\varphi$. By property (ii), $\mathbb{R}_{\geq 1} S$ is a closed convex set in $V$ with boundary $S$ and interior $\mathbb{R}_{>1} S$.

Let $x \in C-\mathbb{R}_{\geq 1} S$. A point $s \in S$ is visible from $x$ if the tangent hyperplane $T_{s} S$ separates $x$ from $\mathbb{R}_{>1} S$. (The tangent hyperplane is being thought of as an affine hyperplane in $V$.) The set $\Omega_{x}:=\{s \in S: s$ is visible from $x\}$ is open in $S$ and invariant by $K(x)=\operatorname{Stab}_{G(\mathbb{R})^{0}}(x)$. If $x=g y$ for $g \in G(\mathbb{R})^{0}$, then $\Omega_{x}=g \Omega_{y}$.

Lemma 1.2. The set of points in $S$ visible from $x \in C-\mathbb{R}_{\geq 1} S$ is relatively compact.

Proof. Let $s=\mathbb{R} x \cap S$. Let $\mathfrak{g}=\mathfrak{k}+\mathfrak{p}$ be the Cartan decomposition given by $s \in C$, let $\mathfrak{a} \subset \mathfrak{p}$ be a maximal abelian subspace, and let $A=e^{\mathfrak{a}}$. Then $x \in(0, s] \subset \mathfrak{a} s$. The set of points visible from $x \in(0, s]$ is $K(x) B$ where $B$ is the set of points in $\mathfrak{a} \cap \cap \mathbb{R}_{>1} S$ visible from $x$ inside the subspace $\mathfrak{a} s$. It suffices to show that $B$ is relatively compact. Using the coordinates on $\mathfrak{a} s$ given by $\mathfrak{a} \cong \mathfrak{a} s$ and the basis $x_{1}, \ldots, x_{r}$ of $\mathfrak{a}$, plus the fact 
that $\mathfrak{a} s \cap S=e^{\mathfrak{a}_{1}} s$, this reduces (by (i) and Lemma 1.1) to the following assertion in $\mathbb{R}^{r}:$ If $B=\left\{\left(t_{1}, \ldots, t_{r}\right) \in\left(\mathbb{R}_{+}\right)^{r}: t_{1}^{\lambda_{1}} t_{2}^{\lambda_{2}} \cdots t_{r}^{\lambda_{r}}=c\right\}$ and all $\lambda_{i}$ are $>0$, then for any $t \in\left(\mathbb{R}_{+}\right)^{r}, t \notin \mathbb{R}_{\geq 1} B$, the set of points on $B$ visible from $t$ is relatively compact. This is easily checked by a direct calculation.

(It is easy to see from the proof that the set of points visible from $x$ is geodesically starlike around the point $\mathbb{R} x \cap S$ and has nonempty interior, and is in fact homeomorphic to a ball.)

1.4. Examples. (i) Let $F / \mathbb{Q}$ be totally real of degree $d$ and $G=\operatorname{Res}_{F / \mathbb{Q}} \mathbb{G}_{m}$, acting on $V_{\mathbb{Q}}=F$. Consider the embedding $F \hookrightarrow V=F \otimes_{\mathbb{Q}} \mathbb{R}=\mathbb{R}^{d}$ given by the various real embeddings of $F$. The cone $C$ is the convex hull of the set of totally positive elements of $F$ in this embedding. The subgroup $G_{1}$ is the norm torus $G_{1}=\operatorname{ker}\left(\operatorname{Res}_{F / \mathbb{Q}} \mathbb{G}_{m} \stackrel{\mathrm{Nm}}{\rightarrow} \mathbb{G}_{m}\right)$, which is $\mathbb{Q}$-anisotropic of rank $d-1$. In suitable coordinates $C \cong \mathbb{R}_{+}^{d}$ and $\varphi\left(t_{1}, \ldots, t_{d}\right)=\left(t_{1} \ldots t_{d}\right)^{-1}$.

(ii) Let $G=\mathrm{GO}(q)$ be the similitude group of a nondegenerate quadratic form $q$ : $V_{\mathbb{Q}} \rightarrow \mathbb{Q}$ such that $q_{\mathbb{R}}$ is of signature $(n, 1)$. The cone $C$ is one component of $\{v \in V:$ $q(v)>0\}, G_{1}=\operatorname{SO}(q)$, and $\varphi(v)=q(v)^{-(n+1) / 2}$ for $v \in C$.

(iii) If $G=\mathrm{GL}(n)$ and $V=\left\{X \in \mathrm{M}(n, \mathbb{R}): X^{t}=X\right\}$ with the action $(g, X) \mapsto$ $g^{t} X g$, then the orbit of the identity matrix is the cone $C$ of positive definite symmetric matrices, equivalently, the set of positive definite quadratic forms in $n$ variables. Here $G_{1}=\operatorname{SL}(n)$ and $\varphi(X)=\operatorname{det}(X)^{-(n+1) / 2}$.

\section{Polyhedral CONE DeCOMPOSITIONS}

In this section polyhedral cone decompositions associated to cocores are introduced (following Ash's Chapter I in [1]) and their top-dimensional cones are enumerated using the notion of visibility.

2.1. Let $V, G, C$ etc. be as in $\S 1$. Let $L \subset V_{\mathbb{Q}}$ be a free $\mathbb{Z}$-module with $L \otimes_{\mathbb{Z}} \mathbb{R}=V$ and let $\Lambda_{0}=\{g \in G(\mathbb{R}): g L=L\}$. A subgroup of $G(\mathbb{R})$ is arithmetic (for the given $\mathbb{Q}$-structure) if it is commensurable with $\Lambda_{0}$. Let $\Lambda$ be an arithmetic subgroup of $G(\mathbb{R})^{0}$ which is neat (i.e. the subgroup of $\mathbb{C}^{*}$ generated by eigenvalues of elements of $\Lambda$ is torsion-free). Let $L^{\times}=L-\{0\}$.

A convex polytope in $V$ is the convex hull of a finite set of points. It is rational if the points are in $V_{\mathbb{Q}}$. A polyhedral cone is a closed convex cone in $V$ of the form $\sigma=\left\{x \in V: \xi_{i}(x) \geq 0\right.$ for $\left.i=1, \ldots, r\right\}$ for some linear functionals $\xi_{1}, \ldots, \xi_{r} \in V^{*}$. It is rational if the linear functionals may be chosen in $V_{\mathbb{Q}}^{*}$. 
2.2. Boundary components. The relation in 1.2 between complete sets of mutually orthogonal Jordan idempotents in $V$ and maximal split tori in $G$ works over $\mathbb{Q}$ : a complete set of mutually orthogonal rational idempotents determines a maximal $\mathbb{Q}$-split torus in $G$ and vice versa (cf. [1, p. 90]). The maximal cardinality of a set of mutually orthogonal rational idempotents, called the $\mathbb{Q}$-rank of $C$, is therefore the same as the $\mathbb{Q}$-rank of $G$. So $C$ has $\mathbb{Q}$-rank one if and only if $\Lambda \mathbb{R}_{+} \backslash C$ is compact.

Let $C_{+}$be the convex hull of $\bar{C} \cap V_{\mathbb{Q}}$. For a rational idempotent $e_{1}$, let $V\left(e_{1}\right)=$ $\left\{x \in V: x \cdot e_{1}=x\right\}$; this is a rational Jordan subalgebra of $V$. The cone of invertible squares $C\left(e_{1}\right)=\left\{x^{2}: x \in V\left(e_{1}\right)\right.$ invertible $\}$ is a self-adjoint homogeneous cone in $V\left(e_{1}\right)$ and $C\left(e_{1}\right) \subset C_{+}$. The cones $C\left(e_{1}\right) \subset C_{+}$as $e_{1}$ varies over rational idempotents are the rational boundary components of $C$. Distinct rational boundary components are disjoint, and the union of all rational boundary components is $C_{+}-\{0\}$ (cf. Remark 3 on p. 133 of [1]). Note that $C_{+}=C \cup\{0\}$ if and only if $C$ has $\mathbb{Q}$-rank one.

\subsection{Examples. Consider the examples in 1.4:}

(i) $G=\operatorname{Res}_{F / \mathbb{Q}} \mathbb{G}_{m}, C \cong \mathbb{R}_{+}^{d}$ the convex hull in $V=F \otimes \mathbb{R}$ of the totally positive elements of $F$. This cone is of $\mathbb{Q}$-rank one and $C_{+}=C \cup\{0\}$. An arithmetic group is a subgroup of the group of totally positive units in the ring of integers of $F$, so if it neat then it is free abelian of rank $d-1$.

(ii) $G=\mathrm{GO}(q)$ and $C$ is a component of $\{q(v)>0\}$. The rational boundary components are half-lines $\mathbb{R}_{+}$, one for each $\mathbb{Q}$-rational $q$-isotropic line in $V$. If there are no such lines $C$ is of $\mathbb{Q}$-rank one; otherwise it is of $\mathbb{Q}$-rank two.

(iii) $G=\mathrm{GL}(n)$ and $C$ the cone of positive definite quadratic forms in $n$ variables. The cone is of $\mathbb{Q}$-rank $n$ (for the standard $\mathbb{Q}$-structure) and $C_{+}$is the set of positive semidefinite quadratic forms in $n$ variables with rational nullspace. An arithmetic group in $\operatorname{GL}(n, \mathbb{R})$ is a group commensurable with $\mathrm{GL}(n, \mathbb{Z})$.

2.4. Kernels, cores, cocores and polarization functions. A closed convex subset $K \subset$ $\bar{C}$ is a kernel if $0 \notin K, C \subset \mathbb{R}_{+} K$, and $\mathbb{R}_{\geq 1} K \subset K$. (So $K$ does not contain 0 and every ray in $C$ is eventually in $K$.) (This is a slight departure from [1], where neither closure nor convexity is required of a kernel.) Two kernels $K_{1}$ and $K_{2}$ are comparable if for some $\lambda_{1}, \lambda_{2} \in \mathbb{R}_{+}$we have $\lambda_{1} K_{1} \subset K_{2} \subset \lambda_{2} K_{1}$.

The closed convex hull of $C \cap L$ is a kernel. Any kernel comparable with it is called a core. (For some other comparable kernels cf. p. 120 of [1].)

The closed convex hull of $\bar{C} \cap L^{\times}$is also a kernel. Any kernel comparable with it is called a cocore.

(Note that if $\mathbb{Q}$-rank $(C)=1$ there is no difference between cores and cocores since $\left.\bar{C} \cap L^{\times}=C \cap L.\right)$ 
There is a duality on kernels which exchanges cores and cocores [1, p. 128]. For a set $K \subset V$ define

$$
K^{\vee}:=\{x \in V:\langle x, y\rangle \geq 1 \text { for all } y \in K\} .
$$

If $K$ is a kernel for $C$ then so is $K^{\vee}$, and $K^{\vee \vee}=K$. If $K$ is a core then $K^{\vee}$ is a cocore.

A kernel $K$ is $\Lambda$-polyhedral if

(i) $\gamma K=K$ for all $\gamma \in \Lambda$

(ii) $K$ is locally rationally polyhedral (l.r.p.), i.e. for any rational polyhedral cone $\Pi$ with vertices in $C_{+}, \Pi \cap K$ is cut out of $\Pi$ by a finite number of supporting hyperplanes of $K$.

The basic examples of such kernels are the closed convex hulls of $C \cap L$ and $\bar{C} \cap L^{\times}$ mentioned above (which are shown to be 1.r.p. on p. 142 of [1]).

If $K$ is a $\Lambda$-polyhedral kernel then $K^{\vee}$ is a $\Lambda^{t}$-polyhedral kernel (cf. p. 141 of [1]). (Here $\Lambda^{t}=\left\{\gamma^{t}: \gamma \in \Lambda\right\}$, an arithmetic group in $G^{t}=G$ ).

A $\Lambda$-invariant polarization function on $C_{+}$is a continuous piecewise-linear function $\phi: C_{+} \rightarrow \mathbb{R}_{\geq 0}$ such that:

(i) $\phi$ is convex: $\phi(x+y) \geq \phi(x)+\phi(y)$ for $x, y \in C_{+}$

(ii) $\phi(x)>0$ if $x \neq 0$

(iii) $\phi$ takes integer values on $C_{+} \cap L$

(iv) $\phi$ is $\Lambda$-invariant

(The notion of convex in (i) is the opposite of the notion of convex used in $\S 1$ for the characteristic function $\varphi$; (i) is the standard convention in the torus embedding game.) Such a function determines a $\Lambda$-polyhedral cocore $P:=\left\{x \in C_{+}: \phi(x) \geq 1\right\}$.

Conversely, given a $\Lambda$-polyhedral cocore $P$, there is a $\Lambda$-invariant polarization function $\phi$ such that $P=\{\phi \geq k\}$ for some large integer $k$. (Define $\phi$ to be the unique convex piecewise-linear function which takes the value $k$ on each face of the cocore and is linear on the cone over each face (cf. p. 310 of [1]). Properties (i), (ii) and (iv) are easy. To get (iii) note that the extreme points of $P$ lie in $\frac{1}{M} L$ for some integer $M[1, \mathrm{p}$. 136]. The lattice generated by these points is of finite index in $\frac{1}{M} L$, so by choosing $k$ suitably we may assume that $\phi$ is integral on $C_{+} \cap \frac{1}{M} L$. Then (iii) holds.) Note that the maximal cones on which $\phi$ is linear are the cones over the faces of $P$.

(For a picture of a cocore $P$ in the case of Example (i) of 1.4 with $F=\mathbb{Q}(\sqrt{3})$ see p. 52 of [1].)

2.5. Facets and visibility. Let $P$ be a closed convex 1.r.p. subset of $V$ of dimension $N$. Recall that a face of $P$ is a subset $f \subset P$ such that every closed line segment whose relative interior meets $f$ lies entirely in $f$. A point $p \in P$ is an extreme point if $\{p\}$ is a face. Let $E(P)$ denote the set of extreme points. A facet of $P$ is a face of dimension 
$N-1$ (facets exist since $P$ is 1.r.p.). For a facet $F$ denote by aff $(F)$ its affine hull; then $\operatorname{aff}(F) \cap P=F$.

Lemma 2.1. Let $P$ be an l.r.p. kernel. The unit inward normal to a facet belongs to $\bar{C}$.

Proof. Let $v_{F}$ be the unit inward (i.e. pointing into $P$ ) normal vector to the facet $F$ of $P$. Suppose that $v_{F} \notin \bar{C}$. Let $\xi: V \rightarrow \mathbb{R}$ be defined by $\xi(x)=\left\langle x, v_{F}\right\rangle$. Then $C-v_{F}^{\perp}$ has two connected components $C_{1}$ and $C_{2}$, where $\left.\xi\right|_{C_{1}}<0$ and $\left.\xi\right|_{C_{2}}>0$. Let $w \in F$. Then $w+v_{F}^{\perp}=\operatorname{aff}(F)$ is a supporting hyperplane of $P$, so $\xi(x) \geq \xi(w)$ for $x \in P$. On the other hand, $\left.\xi\right|_{C_{1}}$ is unbounded below, so that there exists $y \in C_{1}$ such that $\xi(y)<\xi(w)$. Since $\xi(y)<0$, one has $\xi(\lambda y)=\lambda \xi(y) \leq \xi(y)<\xi(w)$ for $\lambda \geq 1$. Thus $\mathbb{R}_{\geq 1} y \cap P=\phi$. But $P$ is a kernel, so any ray in $C$ must eventually be contained in $P$. This is a contradiction, showing that $v_{F} \in \bar{C}$.

For a facet $F$ let aff $(F)_{+}$be the closed affine halfspace containing $P$ and bounded by aff $(F)$. For $x \in V-P$, a facet $F$ of $P$ is visible from $x$ if aff $(F)$ separates $x$ from the interior of $P$, equivalently if $x \notin \operatorname{aff}(F)_{+}$. A facet is relevant if it is visible from 0 . The set of relevant facets is $\Lambda$-invariant. (When the $\mathbb{Q}$-rank of $C$ is one every facet is relevant, cf. Remark 2.7(i) below.)

Lemma 2.2. Let $P$ be a $\Lambda$-polyhedral cocore, $F$ a facet of $P$, and $v_{F}$ its unit inward normal. Then $F$ is relevant if and only if $v_{F} \in C$.

Proof. For any affine hyperplane $H$ meeting $C, \bar{C}-H \cap \bar{C}$ has two components. If either normal to $H$ is in $C$ then exactly one component is bounded, the one containing 0 . Suppose $F$ is irrelevant. Then $\bar{C}-\operatorname{aff}(F)$ has two components, one of which contains 0 in its closure and also contains the interior of $P$. If $v_{F} \in C$ then this component is bounded. But $P$ is unbounded. So $v_{F} \notin C$.

By Remark 1 on p. 132 of [1], if $y \in \bar{C}-C$ then $\inf _{x \in \bar{C} \cap L^{\times}}\langle y, x\rangle=0$. Since $P$ is a cocore, there exists $\lambda \in \mathbb{R}_{+}$such that $P \supset \lambda\left(\bar{C} \cap L^{\times}\right)$, and so $\inf _{x \in P}\langle y, x\rangle=0$. It follows that a facet $F$ with $v_{F} \in \bar{C}-C$ must actually lie in $v_{F}^{\perp}$. But then $0 \in v_{F}^{\perp}=$ aff $(F)$, so $F$ is irrelevant.

(The lemma fails for cores if $\mathbb{Q}$-rank $(C) \geq 2$, as the example on p. 135 of [1] shows. Let $V_{\mathbb{Q}}=\mathbb{Q}^{2}, C=\mathbb{R}_{+}^{2}$ and $P$ the closed convex hull of $\mathbb{Z}^{2} \cap C=\mathbb{Z}_{+}^{2}$. There are facets which are visible from 0 but have normal vector in $\bar{C}-C$.)

Lemma 2.3. Let $H_{+}$be an affine halfspace in $V$ with bounding hyperplane $H$ such that $H \cap C \neq \phi$. Suppose that the inward normal vector $v$ of $H_{+}$belongs to $C$. Then for any $x \in C-H_{+}$the set $\left\{\gamma \in \Lambda: x \notin \gamma H_{+}\right\}$is finite.

Proof. If a sequence of points $x_{i}$ in $C$ tends to a limit in $\bar{C}-C$ then the sequence of values $\varphi\left(x_{i}\right)$ of the characteristic function tends to infinity (Prop. 3 on p. 60 of [1]). Therefore the function $\left.\varphi\right|_{C \cap H}$ extends continuously to $\varphi: \bar{C} \cap H \rightarrow(0, \infty]$ by 
setting $\varphi(x)=\infty$ for $x \in(\bar{C}-C) \cap H$. Since $v \in C$ and $H$ is a translate of $v^{\perp}$, $\bar{C} \cap H$ is compact. So $\varphi$ takes its minimum value at some point $s \in C \cap H$. Since $\varphi$ is strictly convex, this point is unique. The orbit $S=G_{1}(\mathbb{R})^{0} \cdot s=\varphi^{-1}(\varphi(s))$ has tangent hyperplane $H$ at $s$. For $g \in G_{1}(\mathbb{R})^{0}, x \notin g H_{+}$if and only if $g s \in S$ is visible from $x$. The set of such $g s$ is relatively compact by Lemma 1.2, and so the set of such $g$ is relatively compact, i.e. $\left\{g \in G_{1}(\mathbb{R})^{0}: x \notin g H_{+}\right\}$is relatively compact. Since $\Lambda \subset G_{1}(\mathbb{R})^{0}$ is discrete, the intersection $\Lambda \cap\left\{g: x \notin g H_{+}\right\}$is finite.

The following is a key fact:

Lemma 2.4. Let $P$ be a $\Lambda$-polyhedral cocore and $x \in C-P$. The number of facets of $P$ visible from $x$ is finite.

Proof. First let us see that facets visible from $x \in C$ are relevant, i.e. visible from 0 . Let $F$ be visible from $x$, i.e. $x \notin \operatorname{aff}(F)_{+}$. If $F$ is not visible from 0 we have $0 \in \operatorname{aff}(F)_{+}$, in which case $\mathbb{R}_{>1} x \cap \operatorname{aff}(F)_{+}=\phi$ and hence $\mathbb{R}_{>1} x \cap P=\phi$. But $P$ is a kernel, so that every ray in $C$ must eventually be in $P$. So $F$ is visible from 0 (and in fact from any point in the line segment $[0, x]$ ).

Since $P$ is a $\Lambda$-polyhedral cocore, it has finitely many relevant facets modulo the action of $\Lambda$ (Prop. 8 on p. 137 of [1]). Choose representatives $F_{1}, \ldots, F_{r}$ for the $\Lambda$ orbits among these facets. For each $i$, the unit normal vector to $F_{i}$ belongs to $C$ by Lemma 2.2. By Lemma 2.3 the set $\left\{\gamma \in \Lambda: x \notin \gamma\right.$ aff $\left.\left(F_{i}\right)_{+}\right\}$is finite, i.e. only finitely many $\Lambda$-translates of $F_{i}$ are visible from $x$.

2.6. Lemma 2.4 lets us enumerate the relevant facets of $P$ in a nice way using the idea of line shellings from the theory of convex polytopes:

Proposition 2.5. Let $P$ be a $\Lambda$-polyhedral cocore. There is an enumeration $F_{1}, F_{2}, \ldots$ of the relevant facets of $P$ such that for each $k$, the intersection of $F_{k}$ with $\cup_{j<k} F_{j}$ is the union of facets of $F_{k}$ visible from a point in aff $\left(F_{k}\right)$.

Proof. Choose a starting relevant facet $F_{1}$ and a point $x$ in its relative interior. The idea is to move towards 0 along the line segment $(0, x]$ and enumerate the facets as they become visible. From points sufficiently close to $x$ the only visible facet is $F_{1}$. A relevant facet $F \neq F_{1}$, is invisible from $x$ but as one moves towards 0 along the line segment $[x, 0]$ it becomes visible at the point $x_{F}=[x, 0] \cap \operatorname{aff}(F)$. By the previous lemma, the set of points $\left\{x_{F}\right\}_{F}$ is discrete in $[x, 0)$ and accumulates at 0 . Enumerate the facets in the order they become visible, i.e. the order in which one meets the various points $x_{F}$. There is ambiguity only if $x_{F}=x_{F^{\prime}}$ for distinct facets $F$ and $F^{\prime}$. By choosing $x \in F_{1}$ carefully we can ensure that this does not happen, i.e. the points $x_{F} \in[0, x]$ are all distinct as $F$ runs over all facets of $P$. (Indeed, for each pair $F, F^{\prime}$ of facets, the set $\left\{x \in F_{1}: x_{F}=x_{F^{\prime}}\right\}$ is the set of $x$ for which $[x, 0]$ meets aff $(F) \cap \operatorname{aff}\left(F^{\prime}\right)$. This is the projection (centred at 0 ) to $F_{1}$ of a set of dimension $N-2$, hence is of dimension $N-2$. 
As the pair $F, F^{\prime}$ varies, this gives a countable family of $N-2$-dimensional subsets of $F_{1}$, which has dimension $N-1$. Take $x$ outside the union of this family.) This gives an enumeration of the relevant facets. The intersection of $F_{k}$ with $\cup_{j<k} F_{j}$ consists of those facets of $F_{k}$ visible in $\operatorname{aff}\left(F_{k}\right)$ from the point $x_{F_{k}}=[0, x] \cap \operatorname{aff}\left(F_{k}\right)$.

(Note that for each $k$ the union $\cup_{j \leq k} F_{k}$ is homeomorphic to a closed $N-1$-ball: $F_{1}$ is a closed ball, and to go from $\cup_{j \leq k-1} F_{j}$ to $\cup_{j \leq k} F_{j}$ one attaches a closed $N-1$ ball along a subset which is a closed $N-2$-ball in the boundary.)

2.7. Remarks. (i) In the $\mathbb{Q}$-rank one case, every facet of $P$ is relevant. Indeed, in the proof of Lemma 2.2 it was shown that any irrelevant facet $F$ is contained in $\bar{C}-C$. Since $F$ is closed and convex and contains no line, it has at least one extreme point, which is then an extreme point of $P$. The extreme points of $P$ are rational (Prop. 7 on p. 136 of [1]) and nonzero. But $\bar{C}-C$ contains no nonzero rational points if the $\mathbb{Q}$-rank is one.

(ii) In the $\mathbb{Q}$-rank one case, $P^{\vee}$ is a $\Lambda^{t}$-polyhedral (co)core, so that there is an enumeration of its facets as in the proposition.

2.8. Polyhedral cone decompositions. A cocore can be used to construct certain decompositions of $C_{+}$. A $\Lambda$-admissible rational polyhedral decomposition (rpd) of $C_{+}$is a collection $\Sigma$ of rational polyhedral cones in $C_{+}$satisfying

(i) if $\tau$ is a face of $\sigma \in \Sigma$ then $\tau \in \Sigma$

(ii) if $\sigma, \tau \in \Sigma$ then $\sigma \cap \tau$ is a face of $\sigma$ and $\tau$

(iii) if $\sigma \in \Sigma$ and $\gamma \in \Lambda$ then $\gamma \sigma \in \Sigma$

(iv) there are only finitely many cones in $\Sigma$ modulo $\Lambda$

(v) $C=\bigcup_{\sigma \in \Sigma} \sigma \cap C$.

It is simplicial if each $\sigma \in \Sigma$ is a simplicial cone, i.e. the cone on a simplex. It is smooth if

(vi) each $\sigma \in \Sigma$ is generated by a subset of a basis of $L$

and projective if it admits a polarization function, i.e. if

(vii) there is a $\Lambda$-invariant polarization function $\phi$ such that maximal cones on which $\phi$ is linear are precisely the top-dimensional cones of $\Sigma$.

Let us see how a $\Lambda$-polyhedral cocore gives a $\Lambda$-admissible rpd, following Ash. If $F$ is relevant then $v_{F} \in C$, so that $F=\operatorname{aff}(F) \cap P$ is compact and convex, hence equal to the convex hull of its set $E(F)$ of extreme points. Now $E(F) \subset E(P)$, while $E(P) \subset \frac{1}{M} L$ for some $M \in \mathbb{Z}$ (cf. [1, p. 136]), so $E(P)$ is discrete. Then $E(F)$ is finite, $F$ is a convex polytope, and the cone over $F$ is a closed polyhedral cone of dimension $N$. (In contrast the cone over an irrelevant facet has dimension $<N$ and may not be polyhedral.) As $F$ runs over the relevant facets of $P$, the resulting $N$-dimensional polyhedral cones, together with their faces and the cone $\{0\}$, give a $\Lambda$-admissible rpd of 
$C_{+}$, i.e. a collection $\Sigma$ of cones satisfying (i)-(v) of 2.8 (cf. Prop. 8 on p. 137 of [1]). Since the $\Lambda$-polyhedral cocore admits a polarization function, such a $\Lambda$-admissible rpd is necessarily projective, i.e. (vii) holds too.

The $\Lambda$-admissible rpd can be further refined so that (i)-(vii) hold. This is done using barycentric subdivision as follows. It is a consequence of the Siegel property for polyhedral cones (Corollary (i) on p. 116 of [1]) and the neatness of $\Lambda$ that if $\sigma \in \Sigma, \gamma \in \Lambda$ such that $\sigma \cap \gamma \sigma \neq \phi$ then $\gamma$ fixes $\sigma$ pointwise. Fix a set $\left\{\sigma_{i}\right\}$ of representatives for the cones of $\Sigma$ modulo $\Lambda$. For each $\sigma_{i}$ choose a ray $\rho_{i}$ in its relative interior (the barycentre). By the previous observation $\cup_{i} \Lambda \rho_{i}$ is a $\Lambda$-invariant set of barycentres for all cones in $\Sigma$. If the $\rho_{i}$ are chosen (as they can be) so that every cone in the barycentric subdivision of $\sigma_{i}$ with these barycentres is smooth, then the barycentric subdivision of $\Sigma$ using the $\Lambda$-invariant family of barycentres is smooth. Conditions (i)-(v) are unaffected by this procedure and it is easy to see that there is a new polarization function $\phi$ for which (vii) holds. In other words, one can find a $\Lambda$-polyhedral cocore $P$ for which the associated $\Lambda$-admissible rpd $\Sigma$ is smooth and projective. Prop. 2.5 gives an enumeration of the relevant facets of $P$ and hence of the maximal-dimensional cones of $\Sigma$.

A collection of cones $\Sigma$ satisfying (i)-(v) above is locally finite at points of $C$, i.e. each point of $C$ belongs to finitely many cones. (If $x \in C$ belongs to infinitely many cones then by (iv) there exist $\gamma \neq e$ and $\sigma$ with $x \in \sigma \cap \gamma \sigma$. By neatness, $\gamma$ fixes $\sigma$ pointwise, hence fixes $x$. But $\Lambda$ acts freely on $C$.) It is not usually locally finite at points of $C_{+}-C$.

\section{TORUS EMBEDDING}

In this section the cone $C$ will always have $\mathbb{Q}$-rank one. We consider the homological properties of torus embeddings associated to the decompositions of the previous section.

3.1. Torus embeddings. Let us recall some elementary facts and fix some notation about torus embeddings and their topology (cf. e.g. [3]). For the moment $T$ is a torus of dimension $d, \Sigma$ is a fan in $X_{*}(T)_{\mathbb{R}} \cong \mathbb{R}^{d}$ which is locally finite (i.e. every $x \in|\Sigma|-\{0\}$ belongs to finitely many cones of $\sigma$.) We will assume that $|\Sigma|$ has nonempty interior. Assume that $\Sigma$ is smooth, i.e. each cone in $\Sigma$ has a set of generators which extends to a basis for $X_{*}(T)$. For $\sigma \in \Sigma$ let $\operatorname{Star}(\sigma)$ be the union of the relative interiors of cones which intersect $\sigma$. Then $\operatorname{Star}(\sigma)$ is an open neighborhood of $\sigma$ in $|\Sigma|$. Since $\Sigma$ is simplicial, one has $\operatorname{Star}(\sigma) \cap \operatorname{Star}(\tau)=\operatorname{Star}(\sigma \cap \tau)$. The collection $\{\operatorname{Star}(\sigma)\}_{\sigma \in \Sigma}$ is an open cover of $|\Sigma|$. The $\operatorname{Star}(\sigma)$ for $\sigma$ of maximal dimension already cover $|\Sigma|$.

Let $Y:=T_{\Sigma}$ be the torus embedding of $T$ with fan $\Sigma$. The $T$-orbits in $Y$ correspond to cones $\sigma \in \Sigma$. Let $O_{\sigma}$ be the orbit corresponding to $\sigma$ and let $T_{\sigma}$ be the isotropy subgroup along this orbit. If $\bar{T}_{\sigma}$ is the closure of $T_{\sigma}$ in $Y=T_{\Sigma}$ then $\left(\bar{T}_{\sigma}, T_{\sigma}\right) \cong$ $\left(\mathbb{C}^{k},\left(\mathbb{C}^{*}\right)^{k}\right)$ for $k=\operatorname{dim} \sigma$. For $\sigma \in \Sigma$ let $Y(\sigma)$ be the interior of the union of $T$-orbits corresponding to cones in $\operatorname{Star}(\sigma)$. This is an open neighborhood of $O_{\sigma}$ in $Y$ and the 
collection $\{Y(\sigma)\}_{\sigma \in \Sigma}$ is an open cover of $Y$ with the property that $Y(\sigma) \cap Y(\tau)=$ $Y(\sigma \cap \tau)$. The $Y(\sigma)$ for $\sigma$ maximal cover $Y$. For $\sigma$ maximal, the orbit $O_{\sigma}$ is a fixed point, and $Y(\sigma)$ is the union of orbits which have $O_{\sigma}$ in their closure. It is smooth and affine with a single $T$-fixed point and is contractible. The same is true of $Y(\sigma)-T$.

For $\sigma \in \Sigma$, the closure $\bar{O}_{\sigma}$ of the orbit is smooth and projective. Let $\Sigma_{\sigma}$ be the subfan consisting of cones $\tau$ with $\tau \cap \sigma \neq \phi$ and all their faces. The torus embedding $Y_{\sigma}:=T_{\Sigma_{\sigma}}$ is identified with an open neighborhood of $\bar{O}_{\sigma}$ in $Y=T_{\Sigma}$. Choose $\mu: \mathbb{G}_{m} \rightarrow T$ in the relative interior of $\sigma$ and consider $r(y)=\lim _{t \rightarrow 0} \mu(t) y$. Then $Y_{\sigma}=\{y \in Y: r(y) \in$ $\left.\bar{O}_{\sigma}\right\}$ and the morphism $r: Y_{\sigma} \rightarrow \bar{O}_{\sigma}$ is an affine fibration of relative $\operatorname{dimension} \operatorname{dim} \sigma$. In particular, it is a homology isomorphism. For any $T$-stable subvariety $Z \subset \bar{O}_{\sigma}$, the restrictions $r: r^{-1}(Z) \rightarrow Z$ and $r: r^{-1}(Z)-T \rightarrow Z$ are both homology isomorphisms.

(When $\sigma$ is of maximal dimension, $\Sigma_{\sigma}$ is the fan consisting of faces of $\sigma$ and $Y_{\sigma}=$ $Y(\sigma)$.)

3.2. Torus embedding using a polyhedral cone decomposition. Now suppose we are in the following situation: $C \subset V$ is a self-adjoint homogeneous cone of $\mathbb{Q}$-rank one in a vector space $V$ with an integral lattice $L$. The quotient $T:=V_{\mathbb{C}} / L$ is a torus of dimension $N$ with $X_{*}(T)=L$. Let $\phi: C \rightarrow \mathbb{R}_{+}$be a $\Lambda$-invariant polarization function, $P=\{\phi \geq 1\}$ the associated $\Lambda$-polyhedral cocore, and $\Sigma$ the fan consisting of cones on the facets of $P$ and their faces; we assume (cf. 2.8) that $\phi$ is such that $\Sigma$ is smooth.

Let

$$
\begin{aligned}
& Y:=\text { torus embedding of } T \text { using the fan } \Sigma \text { in } X_{*}(T)_{\mathbb{R}}=V \\
& D:=Y-T
\end{aligned}
$$

These schemes are separated and locally of finite type (by the local finiteness of $\Sigma$ at points of $C$ mentioned at the end of 2.8). The scheme $Y$ is smooth while $D$ has normal crossings and each irreducible component is a smooth projective toric variety. There is an action of the arithmetic group $\Lambda$ on each. Since $\Lambda$ is assumed neat it acts freely on $D$ (Indeed, there are $\Lambda$-equivariant homeomorphisms $D / T_{c} \cong P / \mathbb{R}_{\geq 1} \cong C / \mathbb{R}_{+}$and the action on $C / \mathbb{R}_{+}$is free since it is the symmetric space of $G_{1}(\mathbb{R})^{0}$.) The quotient $\Lambda \backslash D$ is projective (an ample line bundle on $\Lambda \backslash D$ can be constructed from the polarization function in the usual way [3, Ch. 3]). The action on $Y$ is not free (every element of $\Lambda$ fixes the identity of $T$ ).

The enumeration $F_{1}, F_{2}, \ldots$ of the relevant facets of $P$ in Prop. 2.5 defines filtrations of $D$ and $Y$ by subvarieties of finite type. Let $\sigma_{1}, \sigma_{2}, \ldots$ be the enumeration of topdimensional cones (i.e. $\sigma_{k}$ is the cone on $F_{k}$ ). For $k \geq 1$ let

$$
\begin{aligned}
\Sigma_{k} & =\text { fan consisting of cones } \sigma_{1}, \ldots, \sigma_{k} \text { and their faces } \\
Y_{k} & =T_{\Sigma_{k}} \text { the torus embedding associated to } \Sigma_{k} \\
D_{k} & =Y_{k}-T .
\end{aligned}
$$


The variety $Y_{k}$ is smooth and of finite type and is identified with an open complex submanifold of $Y$. This defines a filtration $Y_{1} \subset Y_{2} \subset \ldots$ of $Y$ by open subsets with $Y=\bigcup_{k} Y_{k}$. The divisor $D_{k} \subset Y_{k}$ has normal crossing and smooth irreducible components. Each $D_{k}$ is open in $D$ and $D=\bigcup_{k} D_{k}$. Denote the closure of $D_{k}$ in $D$ by $\bar{D}_{k}$; this is a divisor with normal crossings in $Y_{r}$ for $r$ large. The morphisms $\bar{D}_{k} \hookrightarrow \bar{D}_{k+1}$ are closed immersions and $D=\bigcup_{k} \bar{D}_{k}$. Each $\bar{D}_{k}$ is projective (indeed, it admits a finite morphism to the projective variety $\Lambda \backslash D$ which is surjective for $k$ large enough). This gives $D$ the structure of an ind-variety.

Theorem 3.1. The inclusion $D \hookrightarrow Y$ is a homology isomorphism.

Proof. We show by induction on $k$ that for each $k$ the inclusion $D_{k} \hookrightarrow Y_{k}$ is a homology isomorphism. Since $\mathrm{H}_{*}(D)=\lim _{*} \mathrm{H}_{*}\left(D_{k}\right)$ and $\mathrm{H}_{*}(Y)=\lim _{*} \mathrm{H}_{*}\left(Y_{k}\right)$, this will imply the theorem. For $k=1$, the variety $Y_{1}$ is an affine toric variety with a single fixed point, hence is contractible. The same is true of $D_{1}$. Assume that $D_{k-1} \subset Y_{k-1}$ is known to be a homology isomorphism.

For simplicity write $\sigma$ for $\sigma_{k}$ and $F$ for $F_{k}$, the corresponding facet of $P$. By the way in which facets were enumerated, the intersection $F \cap\left|\Sigma_{k-1}\right|$ is the union of facets of $F$ visible from a point in $\operatorname{aff}(F)$. Let $f_{1}, \ldots, f_{r}$ be these facets of $F$. Since $F$ is a simplex, the intersection $f_{0}=f_{1} \cap \cdots \cap f_{r}$ is a face of $F$ of dimension $\operatorname{dim} F-r$, and, moreover, $f_{1}, \ldots, f_{r}$ are exactly the facets of $F$ containing $f_{0}$. Let $\tau_{0} \in \Sigma$ be the cone on $f_{0}$.

Consider the Mayer-Vietoris sequences associated to the coverings $Y_{k}=Y_{k-1} \cup$ $Y_{k}(\sigma)$ and $D_{k}=D_{k-1} \cup D_{k}(\sigma)$ (where $Y_{k}(\sigma)$ is defined as in 3.1 and $D_{k}(\sigma)=Y_{k}(\sigma) \cap$ $\left.D=Y_{k}(\sigma)-T\right)$ :

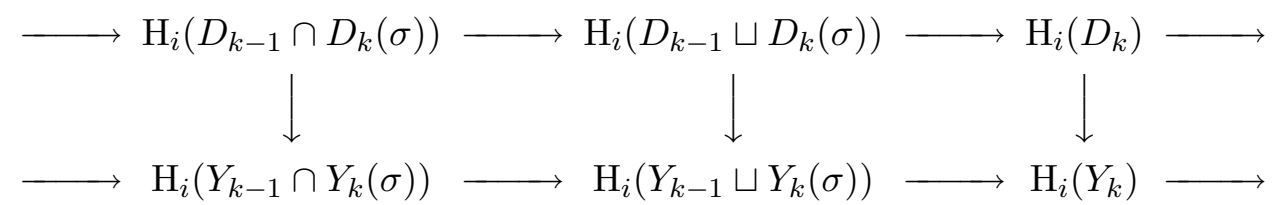

Since $\sigma$ has maximal dimension, $Y_{k}(\sigma)$ and $D_{k}(\sigma)$ are both contractible (a retraction to the fixed point $O_{\sigma}$ being given by any $\mathbb{G}_{m} \rightarrow T$ in the relative interior of $\sigma$.) To complete the inductive step, it suffices to show that $D_{k}(\sigma) \cap D_{k-1} \hookrightarrow Y_{k}(\sigma) \cap Y_{k-1}$ is a homology isomorphism. Let $Z$ be the union of $T$-orbits corresponding to cones on faces $f$ of $F$ such that $f \supset f_{0}$ and $f \subset f_{i}$ for some $i \geq 1$. This is a subset of $\bar{O}_{\tau_{0}}$. Choose $\mu: \mathbb{G}_{m} \rightarrow T$ lying in the relative interior of $\tau_{0}$ and consider the retraction $r: Y_{\tau_{0}} \rightarrow \bar{O}_{\tau_{0}}$. Then $r^{-1}(Z)=Y_{k}(\sigma) \cap Y_{k-1}$ while $r^{-1}(Z)-T=D_{k}(\sigma) \cap D_{k-1}$, so that $r$ gives a retraction of each onto $Z$ which is a homology isomorphism. This completes the inductive step.

3.3. Weights and purity. From now until 3.6 all (co)homology groups and Chow groups have rational coefficients. The rational cohomology of an algebraic variety $X$ carries an increasing weight filtration $\ldots \subset W_{j} \mathrm{H}^{i}(X) \subset W_{j+1} \mathrm{H}^{i}(X) \subset \ldots$ with $\mathrm{Gr}_{j} \mathrm{H}^{i}(X) \neq$ 
$0 \Rightarrow j \in[0,2 i]$ (cf. [2]). The weight filtration on the rational homology $\mathrm{H}_{i}(X)=$ $\mathrm{H}^{i}(X)^{*}$ is the increasing filtration defined by $W_{k} \mathrm{H}_{i}(X)=\left(\mathrm{H}^{i}(X) / W_{-1-k} \mathrm{H}^{i}(X)\right)^{*}$; then $\operatorname{Gr}_{j} \mathrm{H}_{i}(X) \neq 0 \Rightarrow j \in[-2 i, 0]$. If $X$ is proper then $\mathrm{Gr}_{j} \mathrm{H}_{i}(X) \neq 0$ only for $j \geq-i$; if $X$ is smooth then $\operatorname{Gr}_{j} \mathrm{H}_{i}(X) \neq 0$ only for $j \leq-i$. The weight filtration is strictly compatible with the homomorphisms induced by morphisms of varieties, so that one has weight filtrations on $\mathrm{H}_{*}(Y)$ and $\mathrm{H}_{*}(D)$ from the previous subsection.

Corollary 3.2. The homology of $D$ is pure, i.e. $\mathrm{H}_{i}(D)$ is pure of weight $-i$ for each $i$ (and is entirely of Tate type).

Proof. Since each $Y_{k}$ is smooth, $\mathrm{H}_{i}\left(Y_{k}\right)$ has weights $\leq-i$, so $\mathrm{H}_{i}(Y)=\lim _{\longrightarrow} \mathrm{H}_{i}\left(Y_{k}\right)$ has weights $\leq-i$. Since each $\bar{D}_{k}$ is proper, $\mathrm{H}_{i}\left(\bar{D}_{k}\right)$ has weights $\geq-i$, $\overrightarrow{\text { so }} \mathrm{H}_{i}(D)=$ $\lim _{k} \mathrm{H}_{i}\left(\bar{D}_{k}\right)$ has weights $\geq-i$. The isomorphism $\mathrm{H}_{i}(Y) \cong \mathrm{H}_{i}(D)$ respects weights, so this group must be pure of weight $-i$. (That $\mathrm{H}_{i}(D)$ is purely Tate can be seen as follows: Since $D$ has normal crossings, the map $\mathrm{H}_{i}\left(D^{[1]}\right) \rightarrow W_{-i} \mathrm{H}_{i}(D)=\mathrm{H}_{i}(D)$ is surjective, where $D^{[1]} \rightarrow D$ is the normalization (cf. $\left.[2,8.2 .5]\right)$. Since each component of $D^{[1]}$ is a smooth projective toric variety, $\mathrm{H}_{*}\left(D^{[1]}\right)$ is entirely Tate.)

\section{(A slight refinement of this corollary is true, cf. 3.9 below.)}

The theory of weights is not elementary, so it useful to remark that in our context weights have a nice concrete (and elementary) interpretation using natural endomorphisms of toric varieties which lift the various Frobenii, an idea of Totaro [7]. (In the setting of locally symmetric varieties these are the local Hecke operators of [6], cf. 4.1). I briefly sketch his argument to show how it applies here. For each positive integer $n \in \mathbb{Z}_{+}$, the $n$th power map $z \mapsto z^{n}$ on the torus $T$ extends to a finite endomorphism of $Y$ and $D$ preserving each of $Y_{k}, D_{k}, \bar{D}_{k}$ and each irreducible component of each $D_{k}$. Their homology groups become representations of the monoid $\mathbb{Z}_{+}$. Let $X$ be either $Y_{k}$ or $\bar{D}_{k}$. It has a filtration by closed subsets defined by: $X_{i}=$ union of torus orbits of codimension $\geq i$. The corresponding $\mathrm{E}^{1}$ spectral sequence converging to $\mathrm{H}_{*}^{B M}(X)$ is natural with respect to the $\mathbb{Z}_{+}$-action and $n \in \mathbb{Z}_{+}$acts on $\mathrm{E}_{p, q}^{1}$ by multiplication by $n^{p}$. The spectral sequence degenerates at $\mathrm{E}^{1}$, the limit filtration is the weight filtration on $\mathrm{H}_{*}^{B M}(X)$, and the action of $n \in \mathbb{Z}_{+}$on $\mathrm{Gr}_{j} \mathrm{H}_{*}^{B M}(X)$ is by $n^{j}$ (cf. [7, $\left.\S \S 5,6\right]$ ). Now any extension of $\mathbb{Z}_{+}$-representations over $\mathbb{Q}$ of different weights (in the sense that $n$ acts by different powers of $n$ ) has a unique splitting. So the actions of $\mathbb{Z}_{+}$on $\mathrm{H}_{i}\left(Y_{k}\right)=\mathrm{H}_{2}^{B M} \operatorname{dim} Y_{k}-i\left(Y_{k}\right)^{*}\left(Y_{k}\right.$ is smooth) and $\mathrm{H}_{*}\left(\bar{D}_{k}\right)=\mathrm{H}_{*}^{B M}\left(\bar{D}_{k}\right)\left(\bar{D}_{k}\right.$ is proper $)$ are semisimple and split the weight filtration. The same then holds for $\mathrm{H}_{*}(Y)=\lim \mathrm{H}_{*}\left(Y_{k}\right)$ and $\mathrm{H}_{*}(D)=\lim \mathrm{H}_{*}\left(\bar{D}_{k}\right)$. So in fact we could define weights for all our cohomology groups using this action.

Let $D^{[k]}$ be the disjoint union of all $k$-fold intersections of irreducible components of $D$. There is a spectral sequence with

$$
\mathrm{E}_{p, q}^{1}=\mathrm{H}_{q}\left(D^{[p+1]}\right) \Rightarrow \mathrm{H}_{p+q}(D) .
$$


Each $D^{[k]}$ is smooth with projective connected components, so $\mathrm{E}_{p, q}^{1}$ is pure of weight $-q$. The spectral sequence degenerates at $\mathrm{E}^{2}$ and (by definition [2]) the $\mathrm{E}_{2}=\mathrm{E}_{\infty}$ term is $\mathrm{E}_{p, q}^{2}=\mathrm{E}_{p, q}^{\infty}=\operatorname{Gr}_{-q}^{W} \mathrm{H}_{p+q}(D)$. By purity $\mathrm{E}_{p, q}^{2}=0$ for $p>0$, i.e. the rows of the spectral sequence are exact except at the last place. This implies the first part of:

Corollary 3.3. For each $q$ the sequence of $\Lambda$-modules

$$
0 \rightarrow \mathrm{H}_{q}\left(D^{[N]}\right) \rightarrow \cdots \rightarrow \mathrm{H}_{q}\left(D^{[2]}\right) \rightarrow \mathrm{H}_{q}\left(D^{[1]}\right) \rightarrow \mathrm{H}_{q}(D) \rightarrow 0
$$

is a resolution of $\mathrm{H}_{q}(D)$ by finitely generated free $\mathbb{Q}[\Lambda]$-modules.

Proof. We have already seen that the sequence is exact. The connected components of $D^{[k]}$ are parametrized by cones of dimension $k$ in $\Sigma$. Let $\sigma_{1}, \ldots, \sigma_{r}$ be representatives for the $k$-dimensional cones of $\Sigma$ modulo $\Lambda$ and $D_{1}, \ldots, D_{r}$ the corresponding components of $D^{[k]}$; each $D_{i}$ is a smooth projective toric variety and hence has finitedimensional homology. Then $\Lambda\left[\oplus_{i} \mathrm{H}_{q}\left(D_{\sigma_{i}}\right)\right]=\mathrm{H}_{q}\left(D^{[k]}\right)$, so that $\mathrm{H}_{q}\left(D^{[k]}\right)$ is generated over $\mathbb{Q}[\Lambda]$ by a basis for $\oplus_{i} \mathrm{H}_{q}\left(D_{\sigma_{i}}\right)$. The freeness of the $\Lambda$-action follows from the fact that if $\gamma \sigma_{i} \cap \sigma_{i} \neq \phi$ then $\gamma=e$ because $\Lambda$ is neat.

3.4. Homology of $\Lambda \backslash D$. For a space $X$ with a $\Lambda$-action the $\Lambda$-equivariant homology is defined to be

$$
\mathrm{H}_{*}^{\Lambda}(X)=\mathrm{H}_{*}\left(X \times{ }_{\Lambda} E \Lambda\right)
$$

where $E \Lambda$ is any contractible space with a free $\Lambda$-action (in particular, one could take $E \Lambda=C$ or $E \Lambda=V+i C$ ). The Leray spectral sequence for the fibration $X \times{ }_{\Lambda} E \Lambda \rightarrow$ $B \Lambda$ has

$$
\mathrm{E}_{p, q}^{2}=\mathrm{H}_{p}\left(\Lambda, \mathrm{H}_{q}(X)\right) \Rightarrow \mathrm{H}_{p+q}^{\Lambda}(X) .
$$

In the situation at hand, the $\Lambda$-invariance of $\Sigma$ gives an action of $\Lambda$ on the torus embedding $Y$, preserving $D$. Since $D \hookrightarrow Y$ is $\Lambda$-equivariant, the homology isomorphism $\mathrm{H}_{*}(D) \cong \mathrm{H}_{*}(Y)$ of Theorem 3.1 is one of $\Lambda$-modules. By the spectral sequence the induced map $\mathrm{H}_{*}^{\Lambda}(D) \rightarrow \mathrm{H}_{*}^{\Lambda}(Y)$ is also an isomorphism. The differentials in the spectral sequence are natural, in particular they respect weights. By purity, the spectral sequence degenerates at $\mathrm{E}^{2}$. Since $\Lambda$ acts freely on $D, \mathrm{H}_{*}^{\Lambda}(D)=\mathrm{H}_{*}(\Lambda \backslash D)$, giving a natural isomorphism $\mathrm{H}_{*}(\Lambda \backslash D)=\mathrm{H}_{*}^{\Lambda}(Y)$. We arrive at:

Corollary 3.4. There is a natural isomorphism $\mathrm{H}_{*}(\Lambda \backslash D)=\mathrm{H}_{*}^{\Lambda}(Y)$. The spectral sequence $\mathrm{E}_{p, q}^{2}=\mathrm{H}_{p}\left(\Lambda, \mathrm{H}_{q}(D)\right) \Rightarrow \mathrm{H}_{p+q}^{\Lambda}(D)$ degenerates at $\mathrm{E}^{2}$. The limit filtration (suitably shifted) is the weight filtration on $\mathrm{H}_{i}(\Lambda \backslash D)=\mathrm{H}_{i}^{\Lambda}(D)$, so that $\operatorname{Gr}_{-j}^{W} \mathrm{H}_{i}(\Lambda \backslash D)=$ $\mathrm{H}_{i-j}\left(\Lambda, \mathrm{H}_{j}(D)\right)=\mathrm{H}_{i-j}\left(\Lambda, \mathrm{H}_{j}(Y)\right)$.

3.5. Remarks. (i) The analogous results for singular cohomology follow from these results for singular homology since $\mathrm{H}^{*}(Y)=\lim _{k} \mathrm{H}^{*}\left(Y_{k}\right)$. (Our main reason for working in homology rather than cohomology is that it is a little simpler to work with direct limits.) 
(ii) The results, suitably rephrased, hold in $\ell$-adic cohomology. For a discrete group $\Lambda$ acting on a $k$-scheme $X$ the $\Lambda$-equivariant $\ell$-adic cohomology is defined to be

$$
\operatorname{RHom}_{\overline{\mathbb{Q}}_{\ell}[\Lambda]}\left(\overline{\mathbb{Q}}_{\ell}, \mathrm{R} \Gamma\left(X_{\bar{k}}, \overline{\mathbb{Q}}_{\ell}\right)\right) \text {. }
$$

If the action is free, so that $\Lambda \backslash X$ exists as a $k$-scheme, then this is presumably the cohomology $\mathrm{H}^{*}\left((\Lambda \backslash X)_{\bar{k}}, \overline{\mathbb{Q}}_{\ell}\right)$ of the quotient. (In our situation, i.e. $X=D$ and $k=\mathbb{Q}$ this can be deduced from the corresponding statement in singular cohomology using the comparison theorem.) With these definitions, the analogues of the results above in $\mathbb{Q}_{\ell^{-}}$ cohomology hold (and follow from the results for singular cohomology by comparison theorems).

(iii) The results so far also hold if the fan $\Sigma$ is simplicial, provided we work everywhere with rational coefficients.

3.6. Torus-equivariant homology. Another consequence of purity is that the torus action on $D$ is equivariantly formal (cf. [4]). By considerations of weights the spectral sequence for the fibration $D \times_{T} E T \rightarrow B T$ with

$$
\mathrm{E}_{p, q}^{2}=\mathrm{H}_{p}(D) \otimes \mathrm{H}_{q}(B T) \Rightarrow \mathrm{H}_{p+q}^{T}(D)
$$

degenerates at $\mathrm{E}^{2}$ to give $\mathrm{H}_{*}^{T}(D) \cong \mathrm{H}_{*}(D) \otimes \mathrm{H}_{*}(B T)$ (noncanonically). The same holds with $D$ replaced by $Y$, showing, in particular, that $\mathrm{H}_{*}^{T}(D) \cong \mathrm{H}_{*}^{T}(Y)$. The cohomology of the classifying space $\mathrm{H}^{*}(B T) \cong \operatorname{Sym~H}^{2}(B T) \cong \operatorname{Sym} X^{*}(T)$ acts on $\mathrm{H}_{*}^{T}(D)$ by cap product, and $\mathrm{H}_{*}(D)$ can be recovered from $\mathrm{H}_{*}^{T}(D)$ with its $\mathrm{H}^{*}(B T)$-module structure. Since $T$ is a torus, there is a localization of the equivariant cohomology to the $T$-fixed points, and a recipe for computing $\mathrm{H}_{*}^{T}(D)$ in terms of the fixed points and the 1-dimensional torus orbits (the Chang-Skjelbred lemma [4]). All this is natural with respect to $\Lambda$, so that we have, in principle, a description of $\mathrm{H}_{*}(D)$ as a $\Lambda$-module, and hence (by Corollary 3.4) a description of (the associated graded of) $\mathrm{H}_{*}\left(D_{\Lambda}\right)$.

3.7. A refinement of purity. Corollary 3.2 can be improved using the enumeration of the facets of the dual core $P^{\vee}$ mentioned in 2.7(ii). The result, Theorem 3.5 below, will not be used later. We first recall the description of the homology of a smooth projective toric variety using a $\mathbb{G}_{m}$-action (à la Bialynicki-Birula). From now until 3.9 Chow groups and homology groups have integer coefficients.

3.8. Let $T$ be a torus and let $F$ be a convex polytope in $X_{*}(T)_{\mathbb{R}}$ with nonempty interior containing zero. Let $\Sigma_{F}$ be the fan consisting of cones on its facets and their faces, and let $X$ be the torus embedding of $T$ using $\Sigma_{F}$. Assume that $X$ is smooth. Choose $\mu$ : $\mathbb{G}_{m} \rightarrow T$ which lies in the relative interior of a top-dimensional cone of $\Sigma_{F}$. Then the set $X^{\mu\left(\mathbb{G}_{m}\right)}=X^{T}$ of fixed points is finite, in bijection with the top-dimensional cones of $\Sigma_{F}$ or, equivalently, with the facets of $F$. Let us assume that the fixed points have been enumerated $x_{1}, x_{2}, \ldots$ according to visibility of the corresponding facets along the ray through $\mu$. For each fixed point $x_{i} \in X^{\mu\left(\mathbb{G}_{m}\right)}$ define $X_{i}:=\left\{x \in X: \lim _{t \rightarrow 0} \mu(t) x=\right.$ 
$\left.x_{i}\right\}$. Each $X_{i}$ is an affine space and $X_{\leq k}:=\sqcup_{i \leq k} X_{i}$ is closed in $X$ for each $k$. Let $A_{i}(-)$ denote the Chow group of dimension $i$ algebraic cycles on a variety modulo rational equivalence; there is a cycle class map $A_{i}(-) \rightarrow \mathrm{H}_{2 i}^{B M}(-)$ to Borel-Moore homology (i.e. homology with locally finite supports). Let us show by induction that $A_{*}\left(X_{\leq k}\right) \cong \mathrm{H}_{*}^{B M}\left(X_{\leq k}\right)$ for all $k$, the case $k=1$ being trivial. Consider the diagram

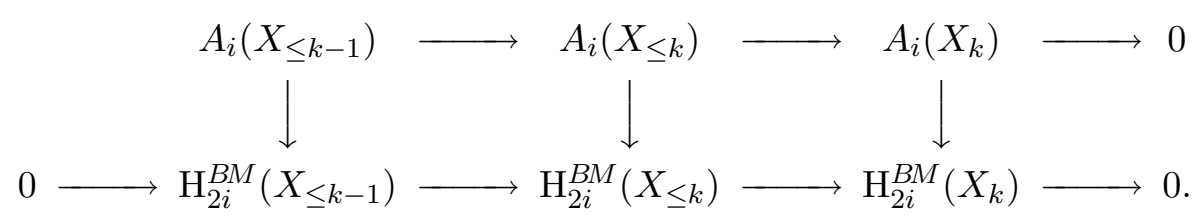

The top row is the usual exact sequence of Chow groups for a closed subscheme and its complement. The bottom row is part of the long exact sequence for Borel-Moore homology; it is short exact since $\mathrm{H}_{i}^{B M}\left(X_{k}\right)$ vanishes in odd degrees. The first and third vertical maps are isomorphisms (by the induction hypothesis and since $A_{*}\left(X_{k}\right) \cong \mathrm{H}_{*}^{B M}\left(X_{k}\right)$ respectively). So $A_{*}\left(X_{\leq k}\right) \cong \mathrm{H}_{*}^{B M}\left(X_{\leq k}\right)$ for all $k$, and hence $A_{*}(X) \cong \mathrm{H}_{*}(X)$ since $X$ is proper.

3.9. Suppose now that we are in the situation of $\S \S 1,2$ and that $C$ has $\mathbb{Q}$-rank one. Let $T_{c} \subset T(\mathbb{C})$ be the compact torus. The quotient $Y / T_{c}$ is naturally identified with $P^{\vee}$; denote the quotient map by $q: Y \rightarrow P^{\vee}$. Let $F_{1}^{\prime}, F_{2}^{\prime}, \ldots$ be the enumeration of the facets of $P^{\vee}$ given by visibility along a line segment $(0, x)$ for $x \in F_{1}^{\prime}$ (cf. Remark 2.7(ii)). For each $k$, let $Z_{k}=\cup_{j \leq k} q^{-1}\left(F_{j}^{\prime}\right)$. Then $Z_{1} \subset Z_{2} \subset \ldots$ is a filtration of $D$ by closed subsets and $D=\cup_{k} Z_{k}$.

Theorem 3.5. The homology of $D$ is concentrated in even degrees, is torsion-free, and is spanned by classes of algebraic cycles.

Proof. It is enough, since $\mathrm{H}_{i}(D)=\lim _{i} \mathrm{H}_{i}\left(Z_{k}\right)$, to prove the corresponding statements for each $Z_{k}$. We will do this by induction. For $k=1, Z_{1}$ is a smooth projective toric variety, so that the cycle class map $A_{*}\left(Z_{1}\right) \rightarrow \mathrm{H}_{*}\left(Z_{1}\right)$ is an isomorphism (by 3.8). The induction hypothesis is that $A_{*}\left(Z_{k-1}\right) \cong \mathrm{H}_{*}\left(Z_{k-1}\right)$. Suppose we knew that

(*) $A_{*}\left(Z_{k}-Z_{k-1}\right) \cong \mathrm{H}_{*}^{B M}\left(Z_{k}-Z_{k-1}\right)$.

Then there is a diagram

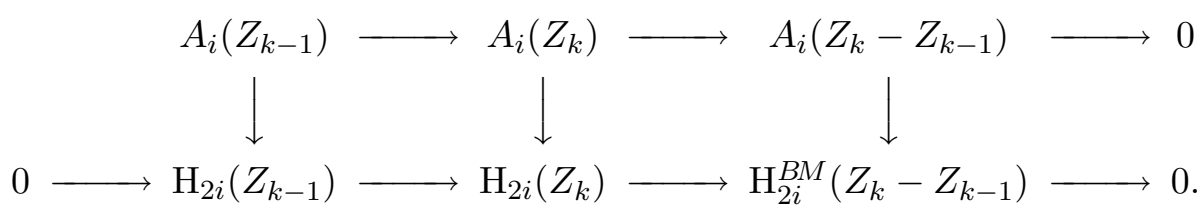

The first row is the usual exact sequence of Chow groups. The second row (part of the long exact sequence in Borel-Moore homology) is short-exact by the induction hypothesis and $(*)$. The first and third vertical maps are isomorphisms, so the second one is too, showing that $A_{*}\left(Z_{k}\right) \cong \mathrm{H}_{*}\left(Z_{k}\right)$. 
The argument to prove $(*)$ uses a suitable $\mathbb{G}_{m}$-action as in 3.8. To simplify notation let $Z^{\prime}:=Z_{k}-Z_{k-1}$ and let $F^{\prime}:=F_{k}^{\prime}$. Let $\sigma \in \Sigma$ be the ray through the vertex of $P$ dual to $F^{\prime}$. The variety $Z^{\prime}$ is open and dense in the smooth projective torus embedding $X$ of the torus $T^{\prime}=T / T_{\sigma}$ associated to the convex polytope $\left(F^{\prime}\right)^{\vee}$. Let $q: X \rightarrow F^{\prime}$ be the quotient by $T_{c}^{\prime}$. Then $X-Z^{\prime}$ is the preimage under $q$ of those facets of $F^{\prime}$ visible from some point $x \in X_{*}\left(T^{\prime}\right)_{\mathbb{R}}$, which may be assumed to be rational. Let $\mu \in X_{*}\left(T^{\prime}\right)$ be a generator of the ray through $x$; consider the $\mathbb{G}_{m}$-action via $\mu: \mathbb{G}_{m} \rightarrow T^{\prime}$. It gives a filtration of $X$ by closed subsets $X_{\leq 1} \subset X_{\leq 2} \subset \ldots$ as in 3.8, with each $X_{k}=$ $X_{\leq k}-X_{\leq k-1}$ an affine space. Since $X-Z^{\prime}$ is closed and $\mu\left(\mathbb{G}_{m}\right)$-stable, we have $X-Z^{\prime}=\bigsqcup_{x_{i} \in X-Z^{\prime}} X_{i}$ and hence $Z^{\prime}=\bigsqcup_{x_{i} \in Z^{\prime}} X_{i}$. Then $Z_{\leq k}^{\prime}:=Z^{\prime} \cap X_{\leq k}$ defines a filtration of $Z^{\prime}$ by closed subsets such that $Z_{\leq k}^{\prime}-Z_{\leq k-1}^{\prime}$ is either empty or an affine space. By the same argument as in 3.8 above, an induction gives $A_{*}\left(Z^{\prime}\right) \cong \mathrm{H}_{*}^{B M}\left(Z^{\prime}\right)$.

3.10. Remarks on the higher-rank situation. We indicate how (some of) the results of this section extend to the situation when $C$ has $\mathbb{Q}$-rank $\geq 2$. No assumption was made in $\S 2$, so the results of that section are general. Thus if $P$ is a $\Lambda$-polyhedral cocore in $C_{+}$and $\Sigma$ the associated rpd. The arguments of $\S 2$ give an enumeration of the facets of the top-dimensional cones in $\Sigma$. As in 3.2 we consider the torus embedding $Y$ along $\Sigma$, and $D=Y-T$. These are no longer locally of finite type, but the schemes $Y_{k}, D_{k}$ are of finite type and the proof of Theorem 3.1 goes through without change, so that $\mathrm{H}_{*}(Y)=\mathrm{H}_{*}(D)$. The statements involving weights also remain valid if we define weights using the action of $\mathbb{Z}_{+}$mentioned after Cor. 3.2, and the same proofs work.

The spectral sequence computing $\mathrm{H}_{*}^{\Lambda}(Y)$ or $\mathrm{H}_{*}^{\Lambda}(D)$ degenerates as before (using weights), but this no longer converges to $H_{*}(\Lambda \backslash D)$ as the action of $\Lambda$ is not free. So the remaining results have to be suitably reformulated.

\section{Arithmetic VARIETIES OF $\mathbb{Q}$-RANK ONE}

In this section we apply the previous results to arithmetic varieties. The notation here differs from that of previous sections. Let $G$ be an algebraic group over $\mathbb{Q}$ such that the symmetric space $D$ of $G(\mathbb{R})^{0}$ is Hermitian. Let $\Gamma \subset G(\mathbb{Q})$ be a neat arithmetic subgroup and $M:=\Gamma \backslash D$ the associated arithmetic variety. Assume that $G$ has $\mathbb{Q}$-rank one, so that $M$ is noncompact. As a notational convention, for subgroups $J \triangleleft H \subset G$, let $\Gamma_{H}:=\Gamma \cap H(\mathbb{R})$ and $\Gamma_{H / J}:=\Gamma_{H} / \Gamma_{J} \subset H / J$.

4.1. Minimal compactification. The compact dual symmetric space $D^{\vee}$ is a flag variety of $G(\mathbb{C})$ with a $G(\mathbb{R})^{0}$-equivariant holomorphic embedding $D \hookrightarrow D^{\vee}$. The boundary components of $D$ are the maximal connected analytic submanifolds of the closure of $D$ in $D^{\vee}$. The stabilizer of a boundary component is a parabolic subgroup whose projection to each simple factor of $G_{\mathbb{R}}$ is a maximal $\mathbb{R}$-parabolic subgroup. The boundary 
component is rational if the stabilizer is $\mathbb{Q}$-rational, in which case it is a maximal $\mathbb{Q}$ parabolic subgroup. The union of rational boundary components $D^{*}$ is $G(\mathbb{Q})$-invariant and carries a natural topology, the Satake topology, for which the $G(\mathbb{Q})$-action is continous. The quotient $M^{*}=\Gamma \backslash D^{*}$ is compact Hausdorff and contains $M$ as an open dense subset. The decomposition of $D^{*}$ into rational boundary components descends to a stratification of $M^{*}$ by complex manifolds, each an arithmetic variety of smaller dimension. There is a unique structure of a normal analytic space on $M^{*}$ restricting to the given complex structure on each stratum. Moreover, according to Baily and Borel, $M^{*}$ is a normal projective variety. Since the $\mathbb{Q}$-rank of $M$ is one and $\Gamma$ is neat, the boundary $M^{*}-M$ is smooth.

4.2. Stabilizer of a boundary component. The stabilizer $P_{F}$ of a rational boundary component $F$ has the following structure (cf. [1, III.4.1-III.4.2]). Its unipotent radical $W$ is two-step unipotent: its centre $U$ is abelian and the quotient $V:=W / U$ is abelian. The quotient $P / W$ splits as an almost-direct product $P / W=M_{\ell} \cdot M_{h}$. (The groups $M_{h}$ and $M_{\ell}$ are denoted $G_{h, F}$ and $G_{\ell, F}$ in [1].) The symmetric space of $M_{h}(\mathbb{R})^{0}$ is the rational boundary component $F$. The adjoint action of $M_{\ell}(\mathbb{R})^{0}$ on $\mathfrak{u}:=\operatorname{Lie} U(\mathbb{R})$ has an open orbit $C \subset \mathfrak{u}$ and the stabilizer of a point is maximal compact. Thus $C$ is a self-adjoint homogeneous cone, of $\mathbb{Q}$-rank one since $G$ is of $\mathbb{Q}$-rank one and $\Gamma_{M_{\ell}}$ is an arithmetic group of its automorphisms (as in $\S \S 1,2$ ).

4.3. Relative torus embedding. Fix a rational boundary component (r.b.c.) $F$. Consider the open domain $D(F):=U(\mathbb{C}) \cdot D$ in $D^{\vee}$, on which $P(\mathbb{R}) U(\mathbb{C})$ acts. Let $T=\Gamma_{U} \backslash U(\mathbb{C})$, a torus of rank $\operatorname{dim} U$, and write $M_{h} W \subset P$ for the preimage of $M_{h}$ by $P \rightarrow P / W$. Let

$$
\begin{aligned}
\mathcal{T} & :=\Gamma_{M_{h} W} \backslash D(F) \\
\mathcal{A} & :=\Gamma_{M_{h} W} U(\mathbb{C}) \backslash D(F) \\
S & :=\Gamma_{M_{h}} W(\mathbb{R}) U(\mathbb{C}) \backslash D(F)=\Gamma_{M_{h}} \backslash F
\end{aligned}
$$

Each is a smooth algebraic variety with a $\Gamma_{M_{\ell}}$-action, the action on $S$ being trivial. There are $\Gamma_{M_{\ell}}$-equivariant morphisms

$$
\mathcal{T} \rightarrow \mathcal{A} \rightarrow S
$$

Here $\mathcal{T} \rightarrow \mathcal{A}$ is a torsor for $T=\Gamma_{U} \backslash U(\mathbb{C})$ and $\mathcal{A} \rightarrow S$ is an abelian scheme with fibres $\mathbb{R}$-isomorphic to $\Gamma_{V} \backslash V(\mathbb{R})$.

Let $\Sigma_{F}$ be a $\Gamma_{M_{\ell}}$-admissible rpd of $C$ which is smooth and projective (as in 2.8). Performing a torus-torsor embedding along the fibres of $\mathcal{T} \rightarrow \mathcal{A}$ using $\Sigma_{F}$ gives a scheme $y \rightarrow \mathcal{A}$ which is locally of finite type over $\mathcal{A}$. Let $\mathcal{D}:=y-\mathcal{T}$. For $s \in S$, write $\mathcal{T}_{s}$ for the fibre of $\mathcal{T} \rightarrow S$ and $\mathcal{D}_{s}:=\mathcal{T}_{s} \cap \mathcal{D}$. Applying Theorem 3.1 fibrewise one concludes that $\mathcal{D}_{s} \hookrightarrow y_{s}$ is a homology isomorphism. The action of $\Gamma_{M_{\ell}}$ on $\mathcal{D}$ is free, and preserves the fibres of $y \rightarrow S$. One gets a natural isomorphism $\mathrm{H}_{*}\left(\Gamma_{M_{\ell}} \backslash \mathcal{D}_{s}\right)=\mathrm{H}_{*}^{\Gamma_{M_{\ell}}}\left(y_{s}\right)$ for 
each $s$. The spectral sequence $\mathrm{E}_{p, q}^{2}=\mathrm{H}_{p}\left(\Gamma_{M_{\ell}}, \mathrm{H}_{q}\left(y_{s}\right)\right) \Rightarrow \mathrm{H}_{*}\left(\Gamma_{M_{\ell}} \backslash \mathcal{D}_{s}\right)$ degenerates at $\mathrm{E}^{2}$ for weight reasons and the statements of 3.4 carry over.

4.4. Toroidal compactifications. Fix a $\Gamma$-admissible rational polyhedral cone decomposition $\Sigma$. Recall what this means: For each r.b.c. $F$ one has a $\Gamma_{M_{\ell}}$-admissible rpd (as in 2.8) $\Sigma_{F}$ of the cone $C$ and the whole collection $\Sigma=\sqcup_{F} \Sigma_{F}$ is required to be $\Gamma$-invariant. Given $\Sigma$, the theory of [1] constructs a toroidal compactification $M^{\Sigma}$. If each $\Sigma_{F}$ is smooth and projective (as in 2.8) then $M^{\Sigma}$ is a smooth projective variety containing $M$ as a Zariski-dense open subvariety, and there is a projective morphism $\pi: M^{\Sigma} \rightarrow M^{*}$ such that $\pi^{-1}\left(M^{*}-M\right)$ is a divisor with simple normal crossings (cf. p. 312 of [1]).

The situation of 4.3 is a local model for the toroidal compactification along the preimage of the stratum $S$ : Fix a stratum $S$ of $M^{*}$ and let $F \subset D^{*}$ be an r.b.c. covering $S$. Let $P, M_{\ell}, M_{h}$ etc. be the associated groups and $\mathcal{D}, y$ etc. the associated schemes. There exist:

(i) a $\Gamma_{M_{\ell}}$-equivariant morphism $y \rightarrow M^{\Sigma}$ (where $\Gamma_{M_{\ell}}$ acts trivially on $M^{\Sigma}$ );

(ii) a neighbourhood $\mathcal{U}$ of $\mathcal{D}$ in $\mathcal{Y}$ (in the classical topology) on which $\Gamma_{M_{\ell}}$ acts freely

such that the induced map $\Gamma_{M_{\ell}} \backslash \mathcal{U} \rightarrow M^{\Sigma}$ is a biholomorphism onto an analytic neighbourhood of $\pi^{-1}(S)$ and restricts to a natural isomorphism

$$
\pi^{-1}(S)=\Gamma_{M_{\ell}} \backslash \mathcal{D}
$$

of complex spaces over $S$. Algebraically speaking, the geometric quotient of the indscheme $\mathcal{D}$ by the action of the discrete group $\Gamma_{M_{\ell}}$ exists and is naturally identified with $\pi^{-1}(S)$ (as a scheme over $S$ ); the formal completion $y_{\mathcal{D}}$ of $y$ along $\mathcal{D}$ has a free action of the discrete group $\Gamma_{M_{\ell}}$, and the geometric quotient is isomorphic to the formal completion $\left(M^{\Sigma}\right)_{\pi^{-1}(S)}$ as a scheme over $S$. The "quotient" $\Gamma_{M_{\ell}} \backslash y$ (which does not exist as a scheme) should be thought of as a formal neighbourhood of $\pi^{-1}(S)$ in $M^{\Sigma}$. Let $\mathcal{D}_{s}$ and $y_{s}$ denote the fibres over $s \in S$. Applying the remarks in 4.3 we arrive at:

Theorem 4.1. For $s \in S$ there is a natural isomorphism

$$
\mathrm{H}_{*}\left(\pi^{-1}(s)\right)=\mathrm{H}_{*}\left(\Gamma_{M_{\ell}} \backslash \mathcal{D}_{s}\right)=\mathrm{H}_{*}^{\Gamma_{M_{\ell}}}\left(y_{s}\right) .
$$

The homology of $y_{s}\left(\right.$ or $\left.\mathcal{D}_{s}\right)$ is pure. The spectral sequence

$$
\mathrm{E}_{p, q}^{2}=\mathrm{H}_{p}\left(\Gamma_{M_{\ell}}, \mathrm{H}_{q}\left(y_{s}\right)\right) \Rightarrow \mathrm{H}_{p+q}^{\Gamma_{M_{\ell}}}\left(y_{s}\right)
$$

degenerates at $\mathrm{E}^{2}$ and the (shifted) limit filtration is the weight filtration on $\mathrm{H}_{*}\left(\pi^{-1}(s)\right)$. The graded pieces are given by

$$
\operatorname{Gr}_{-j}^{W} \mathrm{H}_{i}\left(\pi^{-1}(s)\right)=\mathrm{H}_{i-j}\left(\Gamma_{M_{\ell}}, \mathrm{H}_{j}\left(\mathcal{D}_{s}\right)\right)=\mathrm{H}_{i-j}\left(\Gamma_{M_{\ell}}, \mathrm{H}_{j}\left(y_{s}\right)\right) .
$$


4.5. Remarks. (i) The $\mathbb{Z}_{+}$-action of 3.3 is explicitly realized by Looijenga's local Hecke operators [6] (and the assertion that they split the weight filtration on $\mathrm{H}_{*}\left(\pi^{-1}(s)\right)$ is contained there). One can replace $y_{s}$ by an open neighbourhood of $\mathcal{D}_{s}$ in $y_{s}$ in the classical topology stable under these operators and one can formulate an etale version of the theorem.

(ii) The story in 3.6 carries over to the current context: The $T$-action on $\mathcal{D}$ is equivariantly formal and $\mathrm{H}_{*}^{T}(\mathcal{D})$ is localized to the fixed point locus. The fixed point locus $\mathcal{D}^{T}$ is a disjoint union of copies of $A$, one for each top-dimensional cone. The equivariant homology can be computed from $\mathcal{D}^{T}$ and the locus corresponding to cones of one less dimension, each connected component of which is an $\mathbb{G}_{m}$-fibration over $A$.

4.6. Remark on the Hodge theory of $\pi^{-1}\left(M^{*}-M\right)$. Fix a connected component of $M^{*}-M$ and let $\mathcal{D}, y, \Gamma_{M_{\ell}}$ etc. be as in 4.3. The corresponding component of the exceptional divisor has cohomology

$$
\mathrm{H}_{\Gamma_{M_{\ell}}}^{k}(y, \mathbb{C})=\mathrm{H}^{k}\left(y \times_{\Gamma_{M_{\ell}}} E \Gamma_{M_{\ell}}, \mathbb{C}\right) .
$$

A natural complex computing this group is the total complex associated to the double complex $K^{\bullet \bullet \bullet}=C^{\bullet}\left(\Gamma_{M_{\ell}}, \Gamma(y, \mathscr{E} y)\right)$. (Here $C^{\bullet}\left(\Gamma_{M_{\ell}},-\right)$ is the usual group cohomology complex of a $\Gamma_{M_{\ell}}$-module.) This complex has two filtrations: An increasing filtration $W_{\bullet}$ can be defined by applying the canonical truncation functor $\tau_{\leq i}$ in the first index, a decreasing filtration $F^{\bullet}$ by applying the filtration $F^{p}$ by type to $\mathscr{E} \dot{y}$. It follows from theorems proved above that the spectral sequence for $W_{\bullet}$ degenerates at $\mathrm{E}_{2}$ and the induced filtration on the graded pieces $\operatorname{Gr}_{i}^{W} \mathrm{H}_{\Gamma_{M_{\ell}}}^{k}(y)=\mathrm{H}^{k-i}\left(\Gamma_{M_{\ell}}, \mathrm{H}^{i}(y)\right)$ is the Hodge filtration under the identification $\operatorname{Gr}_{i}^{W} \mathrm{H}_{\Gamma_{M_{\ell}}}^{k}(y)=\operatorname{Gr}_{i}^{W} \mathrm{H}^{k}\left(\Gamma_{M_{\ell}} \backslash \mathcal{D}\right)$. (There is another way to define a naive Hodge filtration on $\mathrm{H}_{\Gamma_{M_{\ell}}}^{k}(y)=\mathrm{H}^{k}\left(y \times_{\Gamma_{M_{\ell}}} E \Gamma_{M_{\ell}}\right)$, namely using the model $E \Gamma_{M_{\ell}}=C+i \mathfrak{u}$, which has a complex structure; this should give the same filtration.)

This suggests that it is possible to understand the mixed Hodge structure (MHS) on the exceptional divisor using the embedding in $M^{\Sigma}$, i.e. it should be possible to find a cohomological mixed Hodge complex (i.e. a complex with two filtrations which define a mixed Hodge structure in cohomology, cf. [2]) consisting of differential forms on a classical neighborhood of $\pi^{-1}(s)$ (of the form $\Gamma_{M_{\ell}} \backslash \mathcal{U}$ for $\mathcal{U}$ as in 4.4) which computes the MHS on $\mathrm{H}^{*}\left(\pi^{-1}(s), \mathbb{C}\right)$. (The Hodge filtration should be the natural one on differential forms. Note that the MHS is defined in [2] without reference to the embedding, and a priori has no simple relation to it, so this is a special feature of arithmetic varieties.) This would then have interesting applications to the Hodge theory of $M^{\Sigma}$, and hence to $M$.

\section{REFERENCES}

[1] A. Ash, D. Mumford, M. Rapoport, Y.-S. Tai, Smooth Compactification of Locally Symmetric Varieties, Math Sci Press, Brookline, MA, 1975. 
[2] P. Deligne, Théorie de Hodge II, Publ. Math. IHÉS 40 (1971), 5-57; Théorie de Hodge III, Publ. Math. IHÉS (44) (1974), 5-77.

[3] W. Fulton, Introduction to Toric Varieties, Ann. of Math. Stud. No. 131, Princeton Univ. Press, Princeton, NJ, 1993.

[4] M. Goresky, R. Kottwitz, and R. MacPherson, Equivariant cohomology, Koszul duality and localization, Invent. Math. 131 (1998), 25-83.

[5] M. Goresky, R. Kottwitz, and R. MacPherson, Homology of affine Springer fibres in the unramified case, Duke Math. J. 121 (2004), 509-561.

[6] E. Looijenga, $L^{2}$-cohomology of locally symmetric varieties, Compositio Math. 67 (1988), 1-20.

[7] B. Totaro, Chow groups, Chow cohomology, and linear varieties, preprint (1998), available at www. dpmms.cam.ac.uk/ ^bt219/papers.html

Arvind N. Nair

School of Mathematics

Tata Institute of Fundamental Research

Homi Bhabha Road

Mumbai 400005, India

E-mail: arvind@math.tifr.res.in 